\title{
Functional Properties of Parietal Visual Neurons: Mechanisms of Directionality Along a Single Axis
}

\author{
B. C. Motter, M. A. Steinmetz, C. J. Duffy, and V. B. Mountcastle \\ The Bard Laboratories of Neurophysiology, Department of Neuroscience, The Johns Hopkins University School of \\ Medicine, Baltimore, Maryland 21205
}

The directional properties of parietal visual neurons (PVNs) were examined using the method of single-neuron analysis in waking monkeys. PVN properties were determined with passive visual stimuli as the animal executed a simple detection task. Parietal area PG was studied in 10 hemispheres of 6 male Macaca mulatta. Each class of parietal neurons was identified in PG: the fixation, projection, visual, and oculomotor neurons; 613 PVNs were identified, 323 were studied quantitatively, and 188 were studied with one or more of the protocols described. The receptive fields of PVNs are commonly large and bilateral, and at the limit some may fill the visual field; for many, the central zone of the visual field is spared when the fields are determined by stimuli that enter from the periphery and transit meridians. The receptive fields vary with the behavioral state, the angle of gaze, and the parameters of the stimuli used to determine them. PVNs are sensitive to stimulus movement but relatively insensitive to stimulus speed; many respond over a speed range of $5^{\circ}-500 \% /$ sec. Stimulus-response relations may be incremental or decremental with increasing speed or show maxima or minima in the midrange of speed, but the response variation over the full range is rarely greater than 2:1. The directional preferences of PVNs with bilateral receptive fields are opponently organized; the preferred directions point either inward toward or outward away from the central line of gaze along the 4 meridians tested, which were equally spaced in the circular dimension of the visual field. The mechanism of the axis directionality of PVNs was studied using conditioning-test paradigms. They revealed a feed-forward inhibition preceding a stimulus, an effect that extends from the leading edge of the stimulus for $10^{\circ}-20^{\circ}$ in front of the moving stimulus and lasts for several hundred milliseconds. A double-Gaussian model of superimposed but unequal excitatory and inhibitory effects suffices to explain the present observations. It places least demand upon the projection of functional properties from the contralateral hemisphere or from the ipsilateral prestriate areas that project upon PG over multistaged pathways and minimal demands upon intracortical processing mechanisms.

The cortex of the inferior parietal lobule of the macaque monkey is a transcortical target of the traditional visual cortical areas and a transthalamic target of the superior colliculus and pretectal

\footnotetext{
Received Mar. 13, 1986; revised May 23, 1986; accepted June 5, 1986.

The research described in this paper was supported by grant 5 R01 EY03168 from the National Institutes of Health, United States Public Health Service.

Correspondence should be addressed to V. B. Mountcastle at the above address.

Copyright $@ 1987$ Society for Neuroscience 0270-6474/87/010154-23\$02.00/0
}

regions of the brain stem. Area PG (Pandya and Seltzer, 1982) of this lobule contains several classes of neurons with different properties, among them one activated by visual stimuli (Motter and Mountcastle, 1981; Mountcastle et al., 1984). Parietal visual neurons (PVNs) are sensitive to moving visual stimuli and to the direction of stimulus motion within their large, often bilateral receptive fields (Motter and Mountcastle, 1981). The stimulus directions optimal for such a neuron commonly differ in different parts of its receptive field; often all those preferred directions are aimed inward toward or outward away from the point of fixation, in opposite directions along the 2 halves of single meridians. The intensities of response differ along different meridians, producing asymmetries in their circular distribution. The present experiments were designed to uncover the mechanism producing these directional properties.

We determined the functional characteristics of PVNs in waking monkeys, with the expectation that knowledge of those properties would allow inferences concerning the role of the parietal system in visuospatial perception. We sought to construct positive images of these perceptual functions, defects which characterize so vividly primates with lesions of the parietal lobe system.

We first give a new description of the receptive fields of PVNs. These receptive fields are dynamically maintained, for their sizes and in-field properties are determined by the behavioral state of the animal at the time of study (Mountcastle et al., 1981); they also vary with the parameters of the stimuli used to establish them. We define the relations between the frequency of discharge of PVNs and stimulus speed and emphasize that, while PVNs are extremely sensitive to stimulus motion, they are relatively insensitive to stimulus speed, even for stimuli moving in the optimal direction.

The directional mechanisms of PVNs were studied by comparing the responses evoked by stimuli moving in opposite directions along a meridian of $100^{\circ}$ extent, centered on the point of fixation (full-field stimuli), with those evoked by stimuli that moved along $40^{\circ}, 20^{\circ}$, and $10^{\circ}$ segments of such an axis. We sought to determine whether the different directionality in the 2 halves of the visual and receptive fields is produced by differences in the local or minute directional properties in different parts of the same single receptive field or by long-range effects. These latter we define as the effects upon response produced by the ordinal sequence in which different parts of the field are engaged by the stimulus. The results show that only rarely can the full-field directional properties be attributed to differences in the local directional properties in different parts of the same single receptive field. Indeed, for almost every cell studied, directionality is largely determined by effects that extend for tens 
of degrees across the receptive field. We present evidence that a feed-forward inhibitory process invades the receptive field of a PVN, extending from the leading edge to $15^{\circ}-20^{\circ}$ in front of a stimulus moving in the "null" direction through the field.

The following paper (Steinmetz et al., 1987) gives the results of an analysis of the full-field, or circular, tuning of the radial selectivities of PVNs, together with an examination of the hypothesis that such a population of neurons could provide signals of the direction of motion of the head and body relative to surrounding visual space or of the direction of motion of environmental objects relative to the position of the head and eyes.

\section{Materials and Methods}

Experiments were made in 10 hemispheres of 6 male Macaca mulatta weighing 3-6 kg. The monkeys were first trained in their cages in a visual detection task. Training was continued in the laboratory, where the animal sat in a quiet enclosure viewing a $127^{\circ} \times 150^{\circ}$ translucent screen. Both the target light, a laser-generated spot of $0.2^{\circ}-0.3^{\circ}$ diameter, and the test stimuli could be presented anywhere within the central $100^{\circ} \times 100^{\circ}$ zone of the tangent screen and moved in any direction at speeds ranging from $5^{\circ}$ to $600 \% \mathrm{sec}$. A behavioral trial began under computer control with presentation of the fixation target, was continued if the animal closed a signal kcy within a certain time, and was terminated if the animal released the key within a limited time after dimming of the target light. Criteria of $90 \%$ correct detections were reached by successive approximations. Test visual stimuli were delivered during the foreperiod of maintained fixation of the target light. Two or three weeks before recording experiments began, silver-silver chloride electrodes were implanted in the orbital rims for electro-oculographic (EOG) recording and a head-fixation device was fixed to the skull, using a sterile operative procedure under general anesthesia. The final training period then began, with head fixation and feedback of eye position. The animal was required to maintain fixation of the target light even though test visual stimuli were back-projected upon the tangent screen and moved through the visual fields, frequently crossing the point of fixation. These test visual stimuli were used in recording experiments to determinc the functional properties of PVNs: They did not control behavior, as did the target light. Correct task performance was assured by using near-threshold dimming electron levels; variable intertrial intervals, intratrial delays to dimming (foreperiods), and target light positions; and trial interruption when fixation deviated, measured by the EOG. The monkey could not predict the time of target light onset or the time to target dimming, and it was required to respond within a limited reaction time. Each successful detection was rewarded with a drop of liquid. Trained animals performed 1000-2000 trials in a training or recording session.

Electrophysiological recording. A recording chamber was attached to the skull above a $19 \mathrm{~mm}$ hole over the posteromedial portion of the inferior parietal lobule, in a second sterile procedure under general anesthesia. Thereafter, recordings were made in the waking state as the animal executed the task described above. The electrical signs of the impulses discharged by cortical neurons were recorded from extracellular positions through Pt-IR glass-coated microelectrodes (Wolbarsht et al., 1960) carried by a Chubbuck microdrive and passed via the hydraulically closed chamber through the intact dura and into the parietal cortex. Axial movement of the electrode was controlled to $1 \mu \mathrm{m}$. Usually, 1 microelectrode penetration was made in each day's recording session of 6-7 hr. After the recording session the cranial chamber was closed, the head fixation was released, and the animal was returned to its living cage overnight. We recorded for an average of $19 \mathrm{~d}$ in each of the 10 hemispheres from which the present data base was drawn. The amplified signals of nerve impulses, the EOG record, and signals of behavioral and stimulus events were fed in parallel to a number of recording and display devices. The sequential time intervals between ncrve impulses, the digitized EOG record (usually at $100 / \mathrm{sec}$ ), and the times of behavioral and stimulus events were stored in files using PDP minicomputers. Simple online and interrun displays and analyses were used to make estimates of the response sensitivity of PVNs to visual stimuli.

Visual stimulation. The background illumination in the test chamber was $1-2 \mathrm{Cd} / \mathrm{m}^{2}$. Test visual stimuli were usually $10^{\circ} \times 10^{\circ}$ bright squares back-projected onto the tangent screen, 0.1-2.0 log units more intense than the background; occasionally, narrow bars were used, and movement was then orthogonal to their long axes. Test stimuli could be moved in any direction on the tangent screen at speeds accurately controlled up to $200 \% \mathrm{sec}$ and less accurately to $600 \% \mathrm{sec}$. The monkey and the tangent screen were viewed with video monitors.

Testing and identification of neurons. Once the action potential of a cortical neuron was isolated and recording appeared stable, a test run was begun. It consisted of visual stimuli moving in randomly sequenced trials in each of 8 directions at $60^{\circ}$ or $90^{\circ} / \mathrm{sec}$ along meridians $100^{\circ}$ long, evenly distributed in the frontoparallel plane, i.e., around the point of fixation. Control classes were also given, one without visual stimulation and another in which a stationary flash was delivered at the point of fixation. Commonly, 6 trials were collected for each class. The action potentials of a neuron under study were displayed as replicas and as radial histograms like that in Figure 3. Every neuron that responded to these stimuli was labeled a PVN and studied in a variety of protocols aimed at determining the distribution of receptive fields, the sensitivity to stimulus speed, the directional preferences along individual meridians, the organization of these axial selectivities in the circular dimension of the visual field, and the mechanisms of those directionalities.

The speed of the stimulus on the tangent screen in our test apparatus was independent of the screen position of the stimulus. There was a substantial error in the apparent speed of the stimulus with increasing eccentricity, as viewed by the monkey. The error approaches $2: 1$ at the extreme eccentricities of $45^{\circ}-50^{\circ}$. Within any single $10^{\circ}$ sector of the screen the change in speed was small. Morcover, whatever changes did occur were identical for full-field and local sector stimuli. Thus, the tangent error for speed did not affect the results of our comparisons between the responses to local sector and full-field stimuli, nor did it affect those of the conditioning-test experiments.

Statistical comparisons were made between the impulse frequencies during the stimulus periods and the prestimulus ongoing activity during the intertrial interval using paired $t$ tests. Simple $t$ tests were used to compare the values between the bins of the response histograms that corresponded to movement of the stimuli in opposite directions through the same zone of space; a probability level of $5 \%$ was the criterion for statistical significance. Peak values of discharge were read from unsmoothed histograms.

A special experimental paradigm was used to study the mechanism of directionality along a single meridian. In any given trial, the $10^{\circ} \times$ $10^{\circ}$ test stimulus was considered either as a full-field stimulus-if it moved through $100^{\circ}$ of the visual field along a meridian - or as a local stimulus - if it moved through $10^{\circ}$ along the same meridian. The local stimuli were centered at 9 different positions along the chosen meridian; both directions were tested in randomly ordered sequences. The average neuronal response rate during the period of stimulation, adjusted for latency, was used as the descriptive statistic. We used a measure of directionality based on one of Thurstone's paired comparison models (Thurstone, 1927) that incorporated both the mean difference and the variance of both responses, expressed as the absolute mean difference between the responses evoked by lights moving in the 2 directions through the same zone of space, $R 1-R 2$, divided by the square root of their summed variances. Thus,

$$
\text { Directional Index }(\mathrm{DI})=(\mathrm{R} 1-\mathrm{R} 2) /\left(S_{\mathrm{R} 1}{ }^{2}-S_{\mathrm{R} 2}\right)^{1 / 2}
$$

We required that the response produced by at least 1 of the stimuli be significantly different from the background at the $5 \%$ level and differ from that mean spontaneous rate by at least 2 SDs of the latter. The DIs for response pairs that satisfied both these requirements were calculated and used to evaluate the observations on directionality. We classified cells with DIs $>1.0$ as displaying directional preference. For our data base a DI of 1.0 corresponds roughly to a value of 0.5 for a DI calculated by dividing the response evoked by stimulus movement in the nonpreferred direction by that evoked in the preferred direction. The incorporation of variance measure in the index, however, had a substantial effect on the relative weighting of directionality above the cutoff value selected.

Anatomical study. Upon the completion of each experiment, the animal was anesthetized and the head was perfused with formalin. Several guide wires were inserted via the microdrive into each hemisphere at locations close to those at which micruelectrode penetrations had been made. Blocks of each hemisphere were cut in the plane of the guide wires, embedded, sectioned at $25 \mu \mathrm{m}$, and stained with thionin. Every section was mounted for study. The sites of entry of microelectrode 
Table 1. Data base for the experiments

\begin{tabular}{lrrrrr} 
Exp. & Pen. & PVN PEN PVNs & Quantity & $\begin{array}{c}\text { Compu- } \\
\text { ter runs }\end{array}$ \\
\hline $89-\mathrm{L}$ & 17 & 10 & 33 & 14 & 70 \\
$89-\mathrm{R}$ & 15 & 4 & 8 & 7 & 34 \\
$90-\mathrm{L}$ & 34 & 21 & 76 & 40 & 273 \\
$90-\mathrm{R}$ & 30 & 14 & 44 & 27 & 143 \\
$91-\mathrm{L}$ & 24 & 18 & 121 & 66 & 449 \\
$91-\mathrm{R}$ & 24 & 18 & 107 & 66 & 397 \\
$92-\mathrm{L}$ & 31 & 12 & 25 & 18 & 82 \\
$93-\mathrm{L}$ & 33 & 19 & 95 & 39 & 156 \\
$94-\mathrm{L}$ & 47 & 16 & 78 & 32 & 175 \\
$94-\mathrm{R}$ & 27 & 9 & 26 & 14 & 70 \\
\hline
\end{tabular}

penetrations were calculated from the reconstruction with an estimated error of $\pm 0.5 \mathrm{~mm}$.

Definitions. The large sizes and bilateral distributions of the receptive fields of PVNs require, for description, several terms, which we define here. The axis of movement is defined as the counterclockwise angle the axis makes with the vertical in the visual field. Frequently axes passed through the point of fixation in the frontoparallel plane containing that point and normal to the central line of gaze and were thus meridians. We define 4 directions of movement along such a meridian: inward toward or outward away from the point of fixation in each half of the meridian. The rotation of such a meridian about the point of fixation within the frontoparallel plane traces out the circular dimension of the visual field within that plane, which here is the tangent screen upon which visual targets and stimuli were projected. Axial directionality refers to the pattern of directional selectivities along a single meridian. The organization of directionalities in the circular dimension refers to variations of the intensity of axial directionality in that circular dimension of the tangent screen, or what Bishop et al. (1980) called axis specificity, or selectivity.

\section{Results}

\section{General description}

\section{The data base}

Neurons of each of the major classes known to occur in the inferior parietal lobule were identified in the present experiments: the fixation/fixation suppression, projection/manipulation, oculomotor, and visual neurons. These classes were described and their encounter rates given in earlier studies (Mountcastle et al., 1975; Lynch et al., 1977). Neurons responsive to visual stimuli during attentive fixation were identified in 141 of the 282 microelectrode penetrations made into the posterior parietal cortex in 10 hemispheres of waking monkeys (Table 1). Six hundred thirteen PVNs were identified in the present series, 323 of them were studied in a quantitative manner, and 188 were put through the protocol required for the classifications of Table 2.

\section{Locations of recordings}

We have abandoned the method of locating microelectrode penetrations by making electrolytic lesions at the time of recording, for we have observed in the parietal homotypical cortex that currents as small as $4 \mu \mathrm{A}$ for $4 \mathrm{sec}$ often result in the abnormal activity or unresponsiveness of neurons within a zone of cortex $1-2 \mathrm{~mm}$ in radius about the site of current passage. These abnormalities may last for days. We identified the site of entry of 118 of the 141 penetrations of Table 1 made into the area of cortex marked on the drawing of the right hemisphere of a monkey brain (Fig. 1, upper right). This zone is included within

\begin{tabular}{|c|c|}
\hline Parameter & Number \\
\hline \multicolumn{2}{|l|}{ Receptive field distribution } \\
\hline Foveal inclusive, bilateral & 113 \\
\hline Foveal sparing, bilateral & 45 \\
\hline Contralateral only & 12 \\
\hline Ipsilateral only & 9 \\
\hline Single axis & 9 \\
\hline Total & 188 \\
\hline \multicolumn{2}{|l|}{ Directionality } \\
\hline Inward & 80 \\
\hline Outward & 24 \\
\hline Mixed & 84 \\
\hline Total & 188 \\
\hline
\end{tabular}

area PG as defined by Pandya and Seltzer (1982). The 7 other outline drawings of Figure 1 are of the lateral surfaces of experimental brains; those of left hemispheres are reversed. The dots mark all the penetrations in which PVNs were identified. Microelectrode penetrations in which other types of parietal neurons were identified were commonly intermingled with those shown in Figure 1 but are not shown here. Over $85 \%$ of the PVNs observed were identified in the penetrations located in Figure 1; the remainder were studied in penetrations we believe to have entered this same region but for which we could not make identifications of the entry sites. PVNs with complex properties occur in other regions of the inferior parietal lobe, particularly in the superior bank of the superior temporal sulcus (Sakata et al., 1980, 1985; Newsome and Wurtz, 1982; Tanaka et al., 1984; Saito et al., 1986).

There is no method that allows accurate identification of the depth of cortical neurons studied at successive positions during microelectrode penetrations of the cortex in waking monkeys. The dural drag, the indentation of the cortex during penetration, and the shift in the relative positions of electrode and cortex between periods of behavioral activity and quiescence all produce large errors. The depth distribution of the neurons we have studied, with depths read from the microdrive, is given in Figure 2. It is clear that, even with the inherent errors, (1) few neurons were studied in the outermost layer, probably II; (2) the distributions are virtually square at $600-2000 \mu \mathrm{m}$ depth, which suggests that recordings were not selective for neurons in any particular layer from III through VI; and (3) 50 neurons were studied at depths indicating they were located in the outermost part of the cortex of the posterior bank of the intraparietal sulcus. However, only 3 of the 50 cells were located at depths below 4000 $\mu \mathrm{m}$, and thus only these 3 may have been in the POa (Seltzer and Pandya, 1978). The functional properties of these 50 neurons did not differ from those of the parent population in any way we could define. We conclude that they, like the larger parent population, were located in PG.

\section{Receptive fields of PVNs}

\section{Neurons with receptive fields that include the central zone of} vision

Sixty percent (113 of 188) of the PVNs we studied subtended receptive fields that included the central zone of vision (Table 2). This group was heterogeneous, for only 22 of 113 of the 


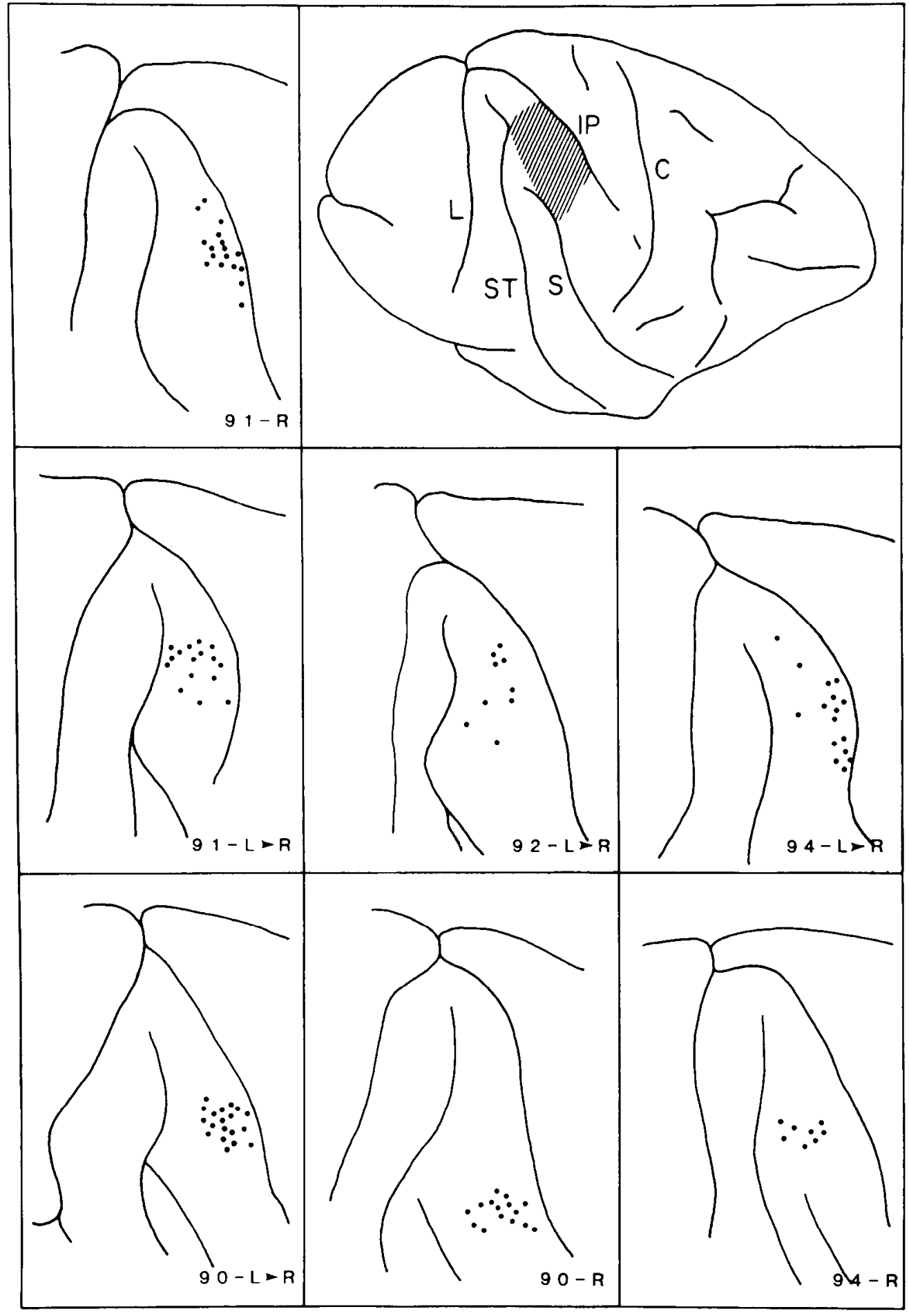

Figure 1. Inset at upper right depicts the lateral surface of the right hemisphere of a monkey, Macaca mulatta. The hatched zone in the inferior parietal lobule includes areas PG and PFG and adjoins the outer strip of POa hidden in the posterior bank of the intraparietal sulcus. Abbreviations: $I P$, intraparietal sulcus; $C$, central sulcus; $S$, sylvian sulcus; $S T$, superior temporal sulcus; $L$, lunate sulcus. The other drawings are of the parietal regions in 7 hemispheres from the experimental group of monkeys; drawings of left hemispheres are reversed. Dots indicate sites of entry of 101 microelectrode penetrations in which PVNs were identified and studied. Parietal neurons of other types were observed in penetrations into this same region (not shown). receptive fields were distributed in a more or less symmetrical way around the point of fixation, with peak responses at that center For the majority ( 91 of 113), the fields and the peak response loci were eccentrically placed, even though the full field extended to include the point of fixation. The results of a study of a neuron with a large, nearly symmetrical receptive field that included the central zone of vision are given in Figures 3 and 4. This PVN was studied $1910 \mu \mathrm{m}$ below the pial surface of area $P G$, just after the microelectrode had been withdrawn from the white matter and reentered the cellular layers of the cortex.
The response replicates, and the radial histograms in Figure 3 show that the neuron was directionally selective, that intense responses were evoked by stimuli moving inwardly along each of the meridians tested, and that the responsive regions extended inwardly far enough to overlap the point of fixation. The activity of this neuron is also graphed in the form of pairs of matched spatial histograms in Figure 4, one of each pair for each direction of stimulus movement along each meridian tested. Spatially comparable bins in each pair of histograms were tested for significant differences. Those significant for direction are marked 


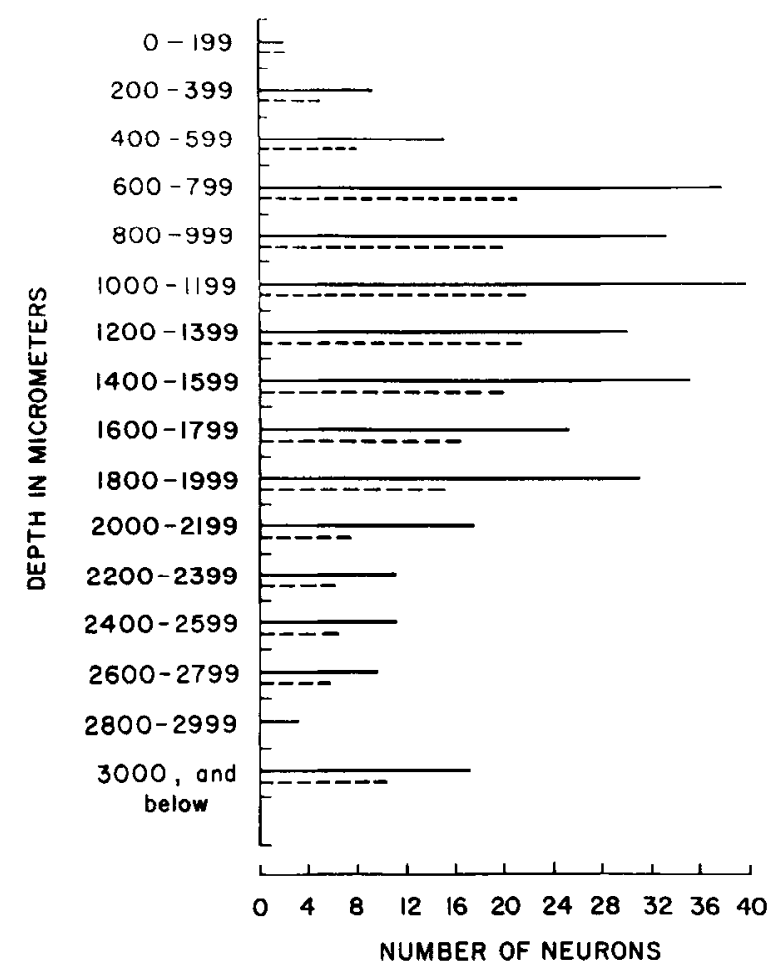

Figure 2. Distribution in depth below the cortical surface of the population of identified PVNs (solid bars) and the smaller number studied in quantitative protocols (dashed bars). Neurons below $2000 \mu \mathrm{m}$ were located in the posterior bank of the intraparietal sulcus, but none was deep enough to be in area POa. Depths read from microdrive; surface of cortex defined as $200 \mu \mathrm{m}$ above the level at which neural activity was first encountered.

with dots ( $5 \%$ level, paired $t$ test). The histograms show that the preferred direction along a meridian changed abruptly as the stimulus moved through a zone $5^{\circ}-6^{\circ}$ in radius, centered on the fixation point. This central zone was sensitive to stationary and moving stimuli but not to the stimulus direction. It is a local region of the receptive field across which the dircctional properties change rapidly.

The radial histograms of Figure 3 show that the responses evoked by inwardly moving stimuli peaked about $10^{\circ}$ away from the point of fixation, thus forming a circle of high intensity at this distance from the central line of vision. The size of the receptive field can be measured from the histograms of Figure $3 \mathrm{C}$. The field diameters along the 4 major axes were $70^{\circ}, 70^{\circ}$, $81^{\circ}$, and $72^{\circ}$. Assuming a circular field, the mean radius of $38^{\circ}$ yields a receptive field area of more than $4500 \mathrm{deg}^{2}$. The receptive field is continuous and extends farther into the ipsilateral than into the contralateral side of the visual field. This neuron is typical of a number of PVNs in its large, bilaterally distributed receptive field, its sensitivity to the movement and direction of movement of stimuli, and the organization of that directionality within the receptive and visual fields. The responses observed are not due to the sudden appearance of the test stimuli ("onset" responses), for we frequently observed that the response at onset increases slowly as stimuli invade the peripheral edge of the receptive fields (see Figs. 3, 15).

Neurons with receptive fields that exclude the central zone of vision

Forty percent of the PVNs we studied were activated from receptive fields that spared the central zone of vision, and of this
$40 \%$ more than half were related to bilateral fields (see Table 2). The degree of central sparing varied continuously from those cells for which only a small area around the point of fixation was unresponsive to those for which the receptive field was confined to narrow rims in the far periphery of the visual field. The receptive fields of 13 PVNs of these types are outlined in Figure 5. In each panel, the central point represents the point of fixation, and the 4 dashed lines the 8 directions ( 2 for each meridian) along which moving stimuli were delivered. Only the central $100^{\circ}$ of the visual field could be tested; thus, the receptive fields of the neurons of Figure $5, A-C$, may have extended beyond the areas shown. Indeed, we have observed, when testing with hand-hcld stimuli during periods of fixation, that many receptive fields of this type extend to the periphery of the visual field. All fields were determined in the manner illustrated in Figure 3; the response areas marked in the panels of Figure 5 are those in which moving stimuli evoked responses significantly greater than the spontaneous rate of impulse discharge of the neuron (5\% level, $t$ test; bin-by-bin analysis as for Fig. 3). We assumed, and intermittent tests confirmed, that the fields were continuous in the regions between the significant response zones along the axes tested.

The receptive field of Figure $5 A$ is bilateral, spares a small central zone, extends to the limits of the visual field we tested, and occupies almost all of the upper half of the visual field. The fields of Figure $5, B-D$, spare large regions of the central zone and are directionally tuned in the opponent manner, either all inward $(A, C)$ or all outward $(B)$ with respect to the point of fixation. The field of Figure $5 D$ is bilateral but eccentrically placed and occupies an intermediate position in the visual field. The 3 fields of Figure $5 E$ are located on the rim of the region tested; all are directionally tuned inward. The 6 fields of Figure $5 F$ are those of cells activated by stimuli moving in only 1 direction along a single axis. Two are located in the ipsilateral half-field, 2 in the contralateral half-field, and 2 were activated by stimuli moving downward along the vertical meridian in the upper half of the visual field.

In summary, the receptive fields of parietal visual neurons appear to fall into 2 classes, those that include the central zone of vision and those that do not, but these 2 may form a continuum. Of the former, only a few (22 of 113) are oriented symmetrically about the fixation point. Among the latter there is a continuous gradient from large fields that exclude only a small central zone to those that occupy a thin rim of space at the periphery of the test. A few receptive fields are much smaller and may be confined to the ipsilateral ( 9 of 188) or the contralateral ( 12 of 188) half of the visual field. There is no correlation between type, size, or distribution of the receptive field of a PVN and its directional properties. We have insufficient data to correlate receptive field type with cortical laminae. The receptive fields of PVNs vary with behavioral state and, as we show in the following section, with certain of the paramctcrs of the stimuli used to determine them.

\section{The variable nature of the receptive fields of PVNs}

The receptive fields of PVNs are labile in size and spatial distribution. A large bilateral field defined during attentive fixation may shrink in size or disappear in an alert but inattentive state (Mountcastle et al., 1981). Such a field may also shrink or enlarge when the fixation target is viewed at a diverted angle of gaze (Andersen and Mountcastle, 1983; Andersen et al., 1984). These changes were observed with test stimuli with constant para- 

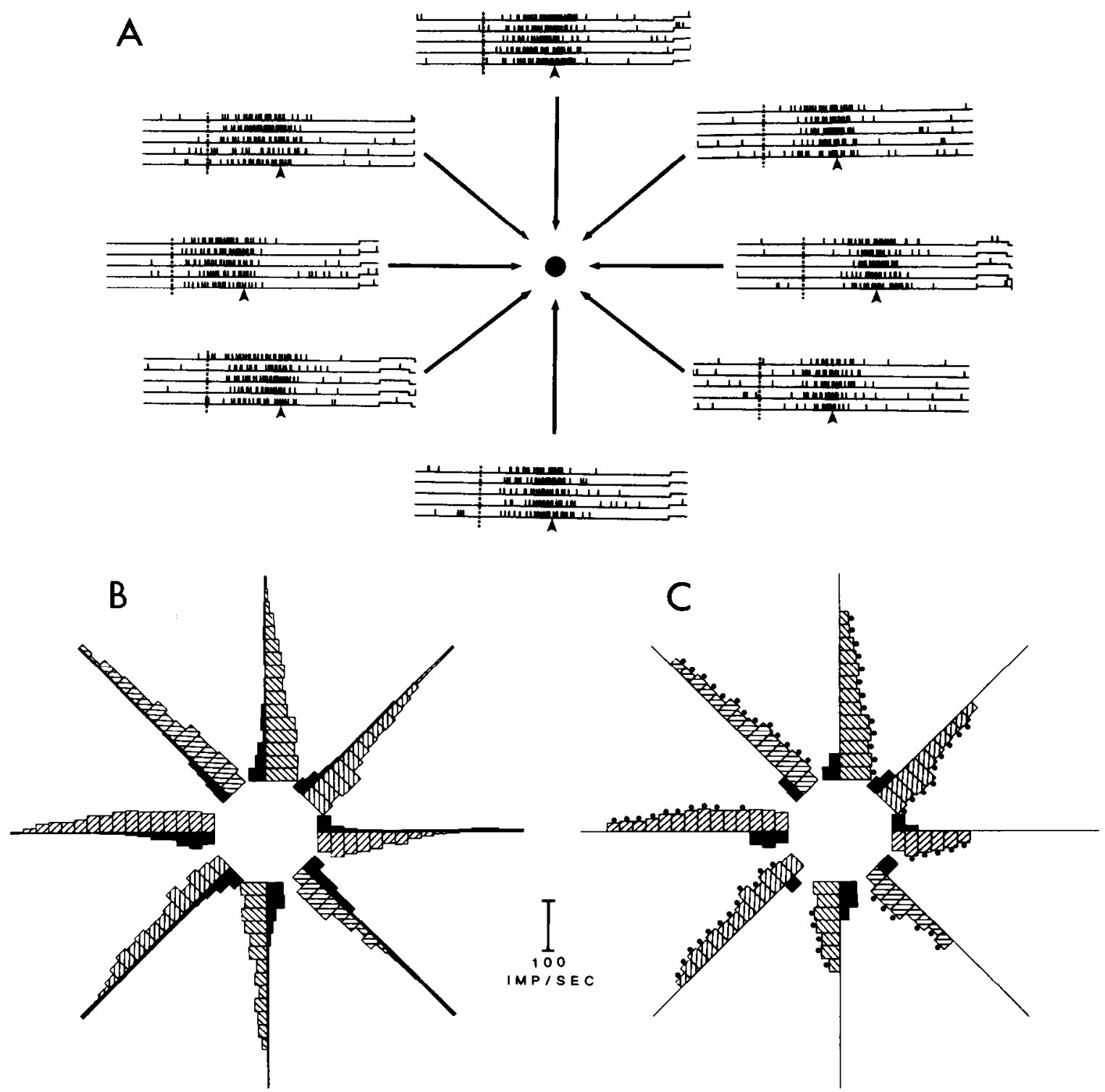

Figure 3. Impulse replicates $(A)$, raw data histograms $(B)$, and histograms of statistically significant responses $(C)$ of a PVN activated by moving visual stimuli. The receptive field of the cell included most of the zone of the visual field tested (a $50^{\circ}$ radius about the fixation point) and the point of fixation. The impulse replicates show the responses (each upstroke the instant of impulse discharge) during repeated trials with a $10^{\circ} \times 10^{\circ}$ visual stimulus moving in each of 8 directions along the 4 meridians tested. Trials in the 8 directions were randomly sequenced. Stimuli were backprojected upon a tangent screen at a $34 \mathrm{~cm}$ viewing distance and moved in the frontoparallel plane for a distance of $100^{\circ}$ centered on the point of fixation. Stimulus speed, $60 \%$ sec. Vertical dashed lines indicate stimulus onset; small arrowheads, the time at which stimuli crossed the fixation point. The radially oriented spatial histograms in $B$ are separated by $20^{\circ}$ in the center for clarity of display. Discharge frequencies during inward halves of stimulus movements are shown by hatching, during outward halves, by solid shading. Bin size is $52 \mathrm{msec}$ and $3.125^{\circ}$; calibration bar, $100 \mathrm{impulses} / \mathrm{sec}$. The histograms in $C$ show the bins in which discharge frequencies were significantly above control levels $(t$ test, $p<0.05)$. Symmetrical bins in the matched histograms were tested for directional preference by paired $t$ test $(p<0.05)$; bins with significant directionality are marked by dots. This neuron responded intensely to both stationary and moving stimuli in a small central zone at the point of fixation in which the directional preference of the neuron reversed, a phenomenon illustrated with greater clarity in Figure 4.

metric values of speed, direction, intensity, size, and shape and thus may be termed dynamic. PVN receptive fields also vary with changes in those stimulus parameters. An example is given in Figure 6. This field was first defined by stimuli moving at $90^{\circ} / \mathrm{sec}$ for $100^{\circ}$ in opposite directions along each of the 4 major meridians; the results for the vertical meridian are shown in Figure $6, A$ and $B$. There is opponent directionality in a field that spares a large central zone. However, the inset histograms of Figure 6, $C$ and $D$, show that physically identical stimuli moving along a $20^{\circ}$ segment of the vertical meridian, centered on the point of fixation and included within the central unre- sponsive zone, evoked intense responses. 'Thus, the extent of the receptive field differed with differences in the history of the stimulus within the visual ficld. Studics of a population of 36 PVNs in which the receptive fields were mapped with both $100^{\circ}$ full-field stimuli and $10^{\circ}$ local segment stimuli revealed a variety of spatial mismatches of the fields mapped in the 2 ways. These included the presence or absence of centrally spared zones, such as the discrepancy of Figure 6, and differences in the lateral extents of the fields. These observations indicate that the field determined by the stimuli of $100^{\circ}$ movement and the strong opponent directionality revealed by these stimuli are deter- 
Figure 4. Statistically reduced histograms shown in Figure $3 C$ are displayed here as pairs, 1 in each pair for the responses evoked by 1 of the 2 directions 4 meridians tested: $A$, vertical; $B$, diagonal, up right $45^{\circ} ; C$, horizontal; $D$, diagonal, up left $45^{\circ}$. Each bin of the spatial histogram equals $3.125^{\circ}$. Directionalities significant at the 0.05 level marked by dots. Central vertical line is the point of fixation. The zone of tranality to its opposite along each meridian occurred in a space no greater than $6.2^{\circ}$ for $A$ and $D$ and $9.3^{\circ}$ for $B$ and $C$. The response patterns of this neuron give an example of strong and balanced opponent organization of directionality along each axis tested. of stimulus movement along each of the sition from one significant direction-

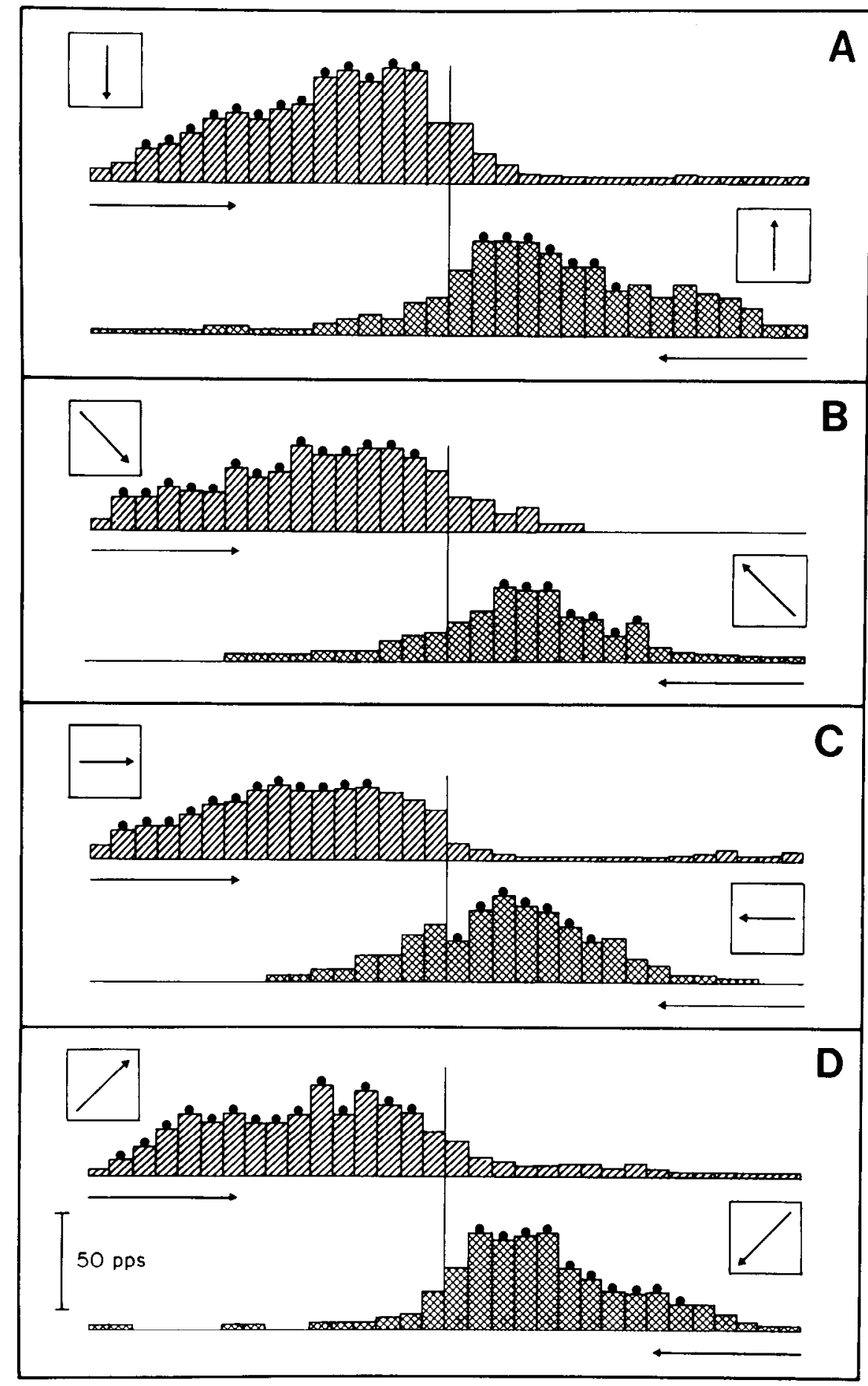

mined by the history of the stimulus within the visual field, a long-range effect we explore further below.

In summary, our present observations and those made in earlier experiments (Motter and Mountcastle, 1981; Mountcastle et al., 1981) suggest that what is to be defined as the receptive field of a PVN is a function both of the behavioral state at the time the field is determined and of the stimulus parameters used for that determination. The receptive fields of PVNs vary in nature and extent; some may occupy the entire visual field. Thus, the function of the parietal component of the visual system may be to provide information not about the exact spatial location of visual stimuli but about motion within and of the surround, the symmetry or asymmetry of that motion, and the spatial relations of objects within the visual field.

Mechanisms of directional selectivity of PVNs along a single meridian of the visual field

Field mismatches like that shown in Figure 6 and opponent directionalities like those shown in Figures 3 and 4 suggested that the full-field directional properties of PVNs are influenced by long-range effects. Our experiments were designed to reveal the properties of this factor. We first compared the directional 

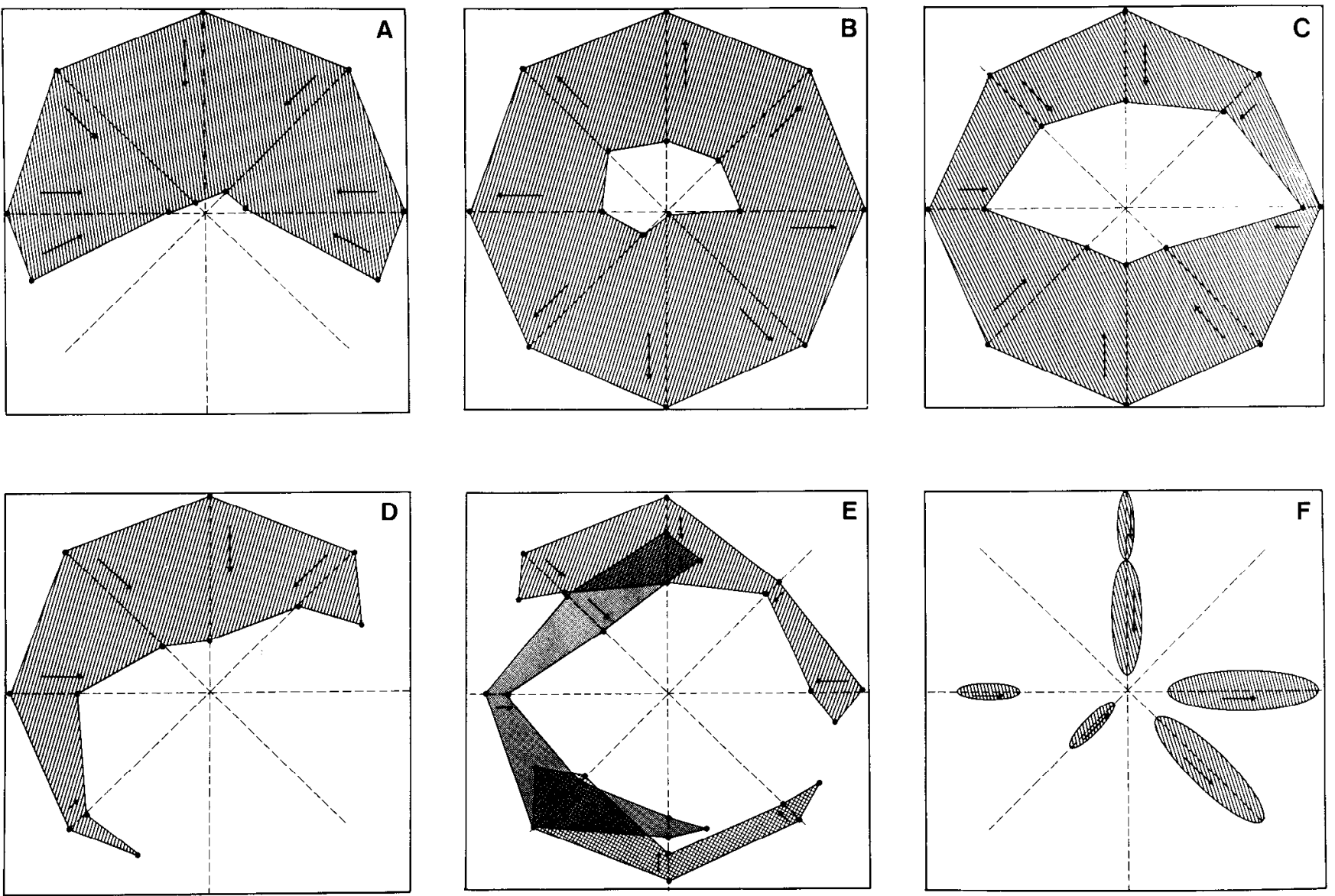

Figure 5. Schematics of the receptive fields of 13 PVNs; all fields spared a central zone of the visual field around the point of fixation. Receptive fields are shown as if the neurons were located in right hemispheres. Neurons were studied and responses analyzed as shown in Figure 3 . Fields were assumed to be continuous between axes along which stimuli evoked responses. $A$, Bilateral field almost confined to the upper half of the visual field, with opponent inward directionality along the horizontal axis. $B$, Bilateral field occupying all of the tested area except for a central zone, with balanced opponent outward directionality. $C$, Bilateral field with large central spared zone, unbalanced opponent inward directionality. $D$, Asymmetrical bilateral field, with inward directionality. $E$. Three receptive fields arranged as narrow rims in the periphery of the visual field; 2 are bilateral and all have inward directionality. $F$, Receptive fields of 6 neurons that responded to moving stimuli in only 1 direction along a single axis; 2 are located in the contralateral visual field and have inward directionality; 2 are related to ipsilateral fields, 1 with inward and 1 with outward directionality; and 2 responded only to stimuli moving downward along the upper half of the vertical meridian.

selectivity along $100^{\circ}$ meridians centered on the fixation point, determined with physically identical stimuli that moved for different distances, in each of the 2 directions through the visual field for (1) full-field stimulus movements covering $100^{\circ}$, (2) stimuli that moved through the lateral $40^{\circ}$ of each half-meridian, and (3) stimuli that moved through $20^{\circ}$ segments at different locations along the meridian. The mismatches between the directional selectivities determined with these stimuli of different spatial extent and those determined with full-field stimuli (see Fig. 3 of the following paper, Steinmetz et al., 1987) confirmed that an effect of considerable spatial extent influences the fullfield directional selectivities of PVNs and that the intensity of this "history" effect varies from strong to weak as the length of stimulus movement decreases. We therefore measured the spatial and temporal properties of this long-range effect, which we found to be a feed-forward inhibitory process.

\section{Directional selectivity in local segments of a meridian} determined with local segment and full-field stimuli

The purpose was to compare the directional selectivity in $10^{\circ}$ segments of a meridian passing through a PVN receptive field,

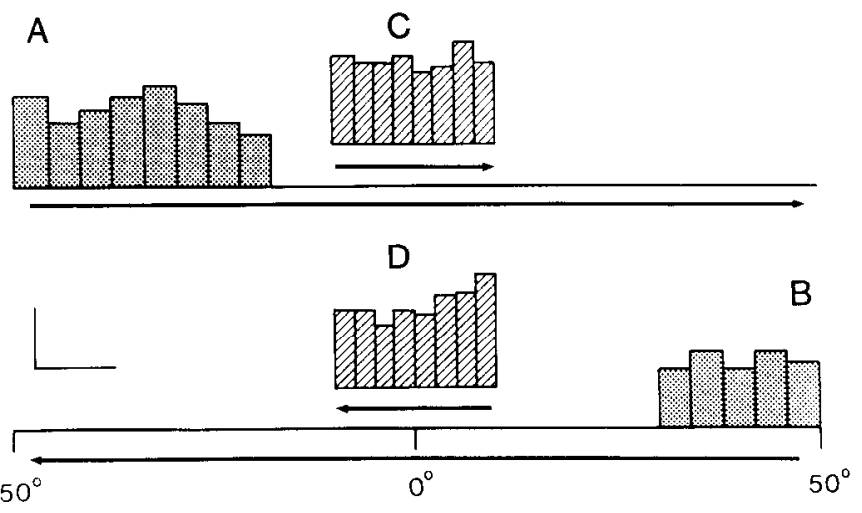

Figure 6. Mismatch of the receptive fields caused by long-range effects of stimulus movement within the field. Histograms $A$ and $B$ show responses evoked by stimuli moving inwardly along a meridian at $90 \%$ sec. The receptive field extended to the edges of the $50^{\circ}$ radius tested but spared a large central zone. Inset histograms $C$ and $D$ show intense but nondirectional responses to identical stimuli moving along $20^{\circ} \mathrm{seg}-$ ments of the same meridian, centered at the point of fixation within the central zone unresponsive to the full-field stimuli. Calibration bars, 50 impulses/sec (vertical); $10^{\circ}$ (horizontal). 


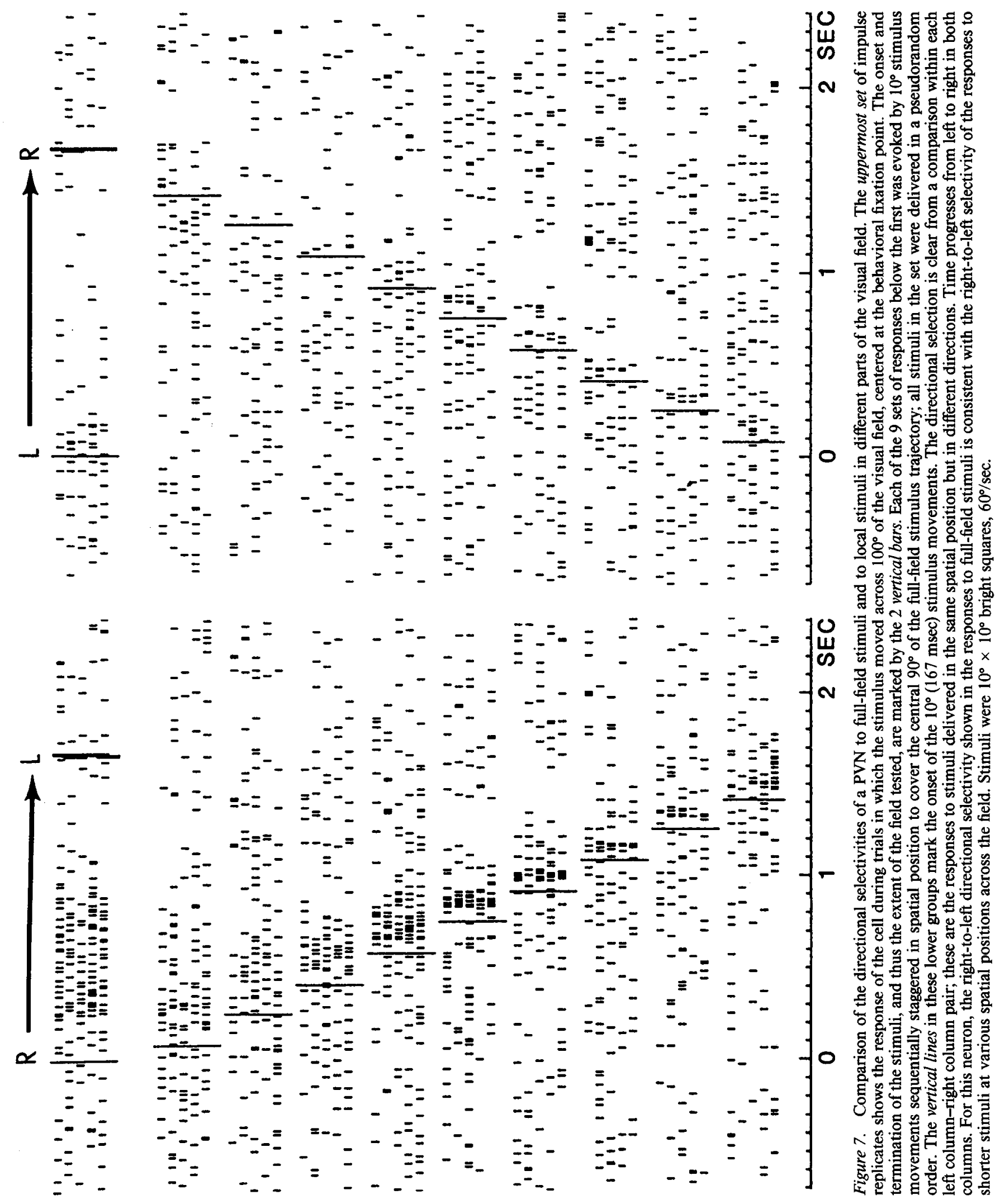


determined in each of 2 ways: by moving stimuli limited to each $10^{\circ}$ segment and by the passage of a full-field stimulus (i.e., of $100^{\circ}$ extent) through each $10^{\circ}$ segment. We chose first to study directional mechanisms along meridians with single, not opponent, directionality in order to isolate the simplest case. For the latter, the null and preferred directions differ in different parts of the meridian; a further study of this more complex situation is under way. We chose, further, to study directional mechanisms in the null direction, because it is in the null direction that responses to local sector and full-field stimuli differ most strikingly. A meridian was chosen for study on the basis of intensity of response and clarity of directional selectivity. The central $90^{\circ}$ of the $100^{\circ}$ meridian was divided into nine $10^{\circ} \mathrm{seg}-$ ments, eliminating the $5^{\circ}$ at either end. Nine local sector stimuli were then presented, each moving through $10^{\circ}$ of visual angle in each of the 2 opposing directions in a random order. The responses were analyzed for significance and directional selectivity as described in Materials and Methods. This study was completed for 25 of 36 neurons selected from the total population of 188 ; for the remaining 11 , testing was made in alternate $10^{\circ}$ local segments. The 36 neurons of the present data base did not differ in any qualitative way from the general population of 188 and included 7 neurons with opponent directional selectivity along some axes.

Figure 7 illustrates the responses of a PVN to the test stimuli delivered. The upper set of impulse replicates (responses) to the right and left shows responses to stimuli moving in opposite directions along the horizontal meridian. The 18 sets of responses of this neuron to local stimuli moving in each of the 2 directions are shown in the 9 pairs of response sets below. Responses to the 2 stimuli delivered in the same local sector are arranged in horizontal pairs, each appropriately located relative to the full-sweep responses above. These response pairs show what analysis confirmed, that the directional selectivity for leftward-moving full-field stimuli is also present in each paired set of responses to local sector stimuli. The neuron of Figure 7 was 1 of only 3 in the set of 36 studied in this way for which this was true. When the receptive fields were defined by including all sectors that responded to either full-field or local sector stimuli, such a match for directionality between full-field and local sector tests was present in only $33 \%$ of the local sectors within the receptive fields. The presence of directionality in about one third of the local sectors tested is strong evidence that the responses we have observed are not "onset" responses. Indeed, we have observed directionally tuned responses in such local sectors using stimuli that moved only $1^{\circ}$ in either direction.

The neuron of Figure 8 illustrates a set of responses typical of the majority of PVNs studied in this way. The strong directional preference for stimuli moving upward along the lower left-upper right diagonal meridian is shown by the upper sets of responses. The corresponding pairs of responses to local sector stimuli, arranged here like those of Figure 7 , are strikingly different; for them the DIs reached criterion value in only 2 of the 7 local sectors of the receptive field. We conclude that the absence of responses to full-field stimuli moving in the null direction is largely determined by a long-range effect. The dominance of long-range effects in determining full-field directional sensitivity along a single meridian has been observed for neurons with all types of field distributions and directional organizations, including those with opponent organization. How unpredictable the strength of directionality determined with local sector stimuli is compared with that determined from full- field stimuli is illustrated by the graph of Figure 9. For some local sectors the directionality was stronger when tested with local stimuli than with full-field stimuli, thus accounting for the points above the diagonal in Figure 9, even though local directionality seldom accounted for the full pattern of directionality shown with full-field stimuli.

\section{Influence of long-range effects in determining the directional selectivity of $P V N s$}

We studied these spatial and temporal interactions further with conditioning-test paradigms in a subset of 14 of the 36 neurons described in the last section. Neurons chosen were those for which local slimuli evoked vigurous responses and for which the full-field stimuli revealed a strong directional preference, with no response at all for stimuli moving in the null direction. The latter was chosen as the test direction. These neurons are therefore not cells with opponent directionality along the axes studied.

The observations made in the conditioning-test experiments are illustrated for a neuron for which each of the 3 paradigms was completed; it is the directionally sensitive neuron of Figure 8 . The paradigm of Figure 10 was used to test the normal spatial and temporal relations between the responses evoked in successive small sectors traversed by full-field stimuli, using smallsector stimuli to mimic the relations traced in space by the fullfield stimuli. The meridian chosen for study was the lower left (LL) to upper right (UR) diagonal meridian. The fixation point was shifted $20^{\circ}$ in the LL direction along this diagonal to bring as much as possible of the contralateral part of the receptive field onto the tangent screen. The upper set of replicates in Figure 10 shows that no significant responses were evoked by a stimulus moving in the null direction for $100^{\circ}$ through the visual field, from $30^{\circ} \mathrm{LL}$ to $70^{\circ} \mathrm{UR}$. The second set of records shows the intense responses to $10^{\circ} \mathrm{small}$-sector stimuli centered $30^{\circ} \mathrm{UR}$ from the fixation point and moving in the null direction; i.e., that in which the full-field stimuli elicited no responses at all. The lowest set of records shows fixation control trials in which no stimuli were delivered. For the third through the seventh sets of records, conditioning stimuli were delivered in 5 spatial locations "proximal" to that of the test stimulus, in a random sequential order from trial to trial, and appropriately timed with respect to the test stimuli to mimic the normal times and sequential spatial positions occupied by the visual stimuli during full-field movements. The conditioning and test stimuli each moved at $60 \% \mathrm{sec}$ and lasted for $167 \mathrm{msec}$. The records of Figure 10 show that the responses to test stimuli were reduced by conditioning stimuli delivered within the two $10^{\circ}$ sectors adjacent to the test sector but not by the 3 delivered in more distant sectors. This suggests that a long-range, feed-forward inhibitory process had a spatial extent of at least $15^{\circ}-20^{\circ}$ in the visual field for this cell. The conditioning stimuli in this case evoked responses in sectors in which the full-field stimuli evoked none. We have frequently observed in other neurons, however, that stimuli may be effective conditioning agents when they themselves evoke no responses from the neuron under study (e.g., Fig. 12). The suppressive effects of full-field stimuli moving in the null direction vary from the present case, in which the suppression affects the evoked response but not the spontaneous activity, to others, in which both the evoked and spontaneous activity are completely suppressed. This experiment was completed for 7 neurons, of which 6 showed results comparable to those of Figure 10, as shown by the graph in Figure $13 \mathrm{~A}$. 


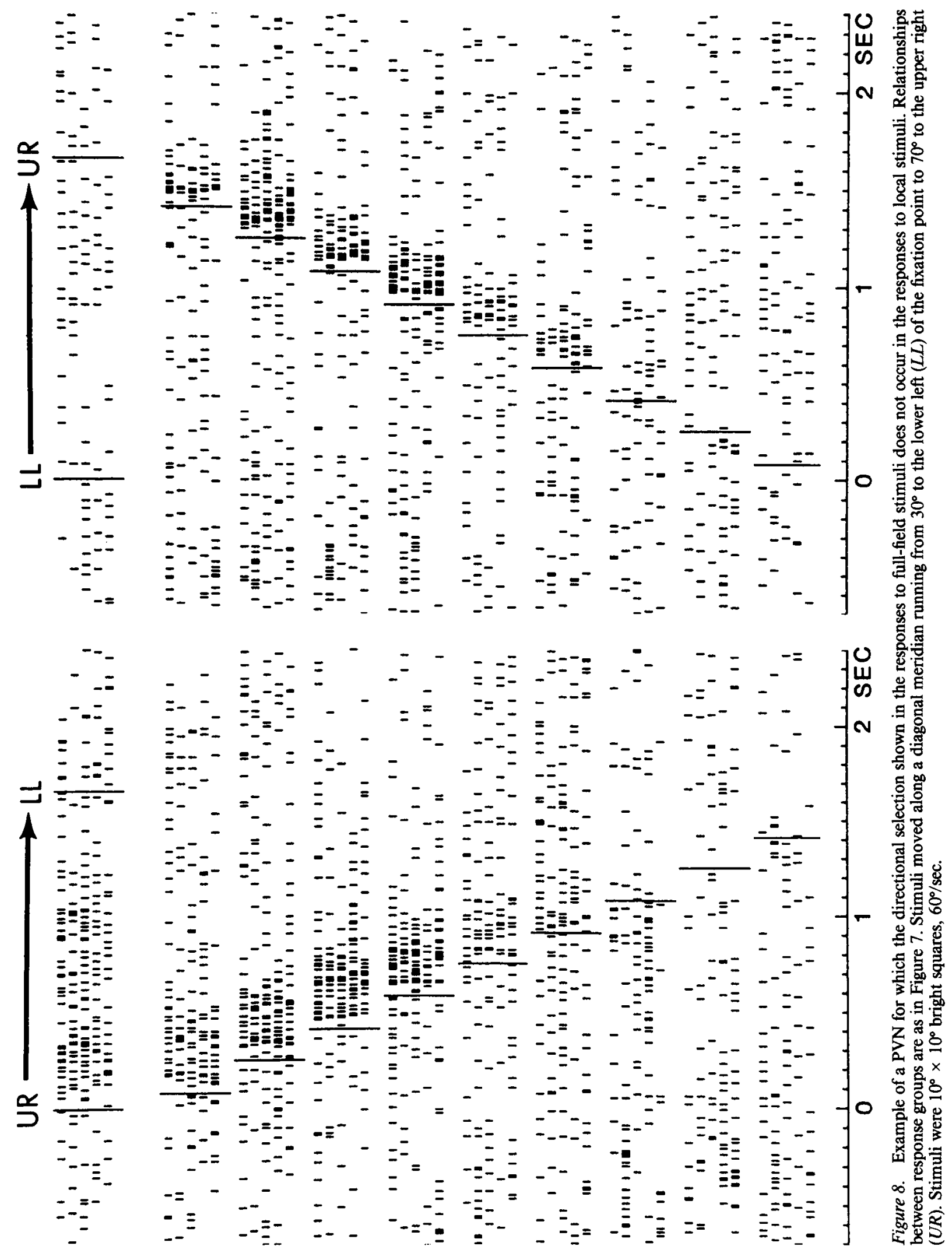


A second paradigm was used to dissociate the spatial and temporal aspects of the feed-forward inhibitory process. The third through the seventh sets of records of Figure 11 were obtained in a series in which the conditioning stimuli were always placed in the $10^{\circ}$ local sector immediately adjacent to the test sector, but the lead time between the 2 stimuli varied over the range required for a full-field movement. Stimuli at the different lead times were sequenced randomly. It is clear that conditioning stimuli suppressed the test response over the $667 \mathrm{msec}$ tested and that the suppressive effect increased in intensity as the conditioning-test interval decreased. The eighth through the tenth sets of records in Figure 11 were obtained in a series in which the time of conditioning stimulus onset was held constant, leading the test stimulus by $250 \mathrm{msec}$. The 3 spatial locations in which conditioning stimuli were delivered corresponded to the first, middle, and last conditioning locations used in the paradigm of Figure 10 . The records show that only the stimulus in the spatial segment immediately adjacent to that of the test stimulus effectively suppressed the test response. The 2 series illustrated in Figure 11 indicate that spatial factors are more critical than temporal factors in governing the suppression of test responses. This is consistent with the broad range of speed sensitivity of PVNs described below.

A third paradigm was devised to define the spatial extent of the inhibitory process more clearly. The results obtained with it for the neuron of Figures 10 and 11 are shown in Figure 12. For the 3rd through the 6th sets of records in Figure 12, the conditioning stimuli were delivered at appropriate positions and lead times relative to the test stimuli, and their spatial movements were successively extended towards the test sector. The test stimulus was always $10^{\circ}$ in spatial extent. The results illustrate that (1) the conditioning stimulus need not elicit a response to be effective in suppressing the response to the test stimulus; (2) the conditioning stimulus was most effective when it invaded the segment of space adjacent to that of the test stimulus; (3) passage of the conditioning stimulus through more distant spatial locations also produced suppressive effects; and (4) the suppressive effect weakened moderately as the conditioning stimuli were shortened from the LL end while the position of the UR end was held at the point $5^{\circ}$ away from the test sector. The average rates of discharge in the responses to the test stimuli were $51 \mathrm{impulses} / \mathrm{sec}$ for the test stimulus alone and $54,47,38$, $14,11,18$, and 16 impulses/sec for the conditioning-test series, from top to bottom. These observations confirm in different ways those of Figure 10. They indicate that the feed-forward inhibitory effect may extend for $15^{\circ}-20^{\circ}$ in front of the full-field stimulus moving in the null direction along a directionally selective meridian. This experiment was made on 11 PVNs; of these, 8 showed effects similar to that of Figure 12, and 1 showed no effect (see Fig. 13, B, C). Two other cells showed a modest facilitation as the conditioning stimuli approached the location of the test stimuli.

In summary, the results of the conditioning-test experiments define some of the spatial and temporal properties of the feedforward inhibitory process that plays a role in producing the directional selectivity of PVNs. The process is evoked by stimuli moving in the null direction, may extend in space up to $20^{\circ}$, and persists for more than $0.6 \mathrm{sec}$. When evoked by local stimuli, the process may suppress responses in an immediately adjacent test sector but not responses evoked within the conditioning sector itself-further evidence for its feed-forward nature. The

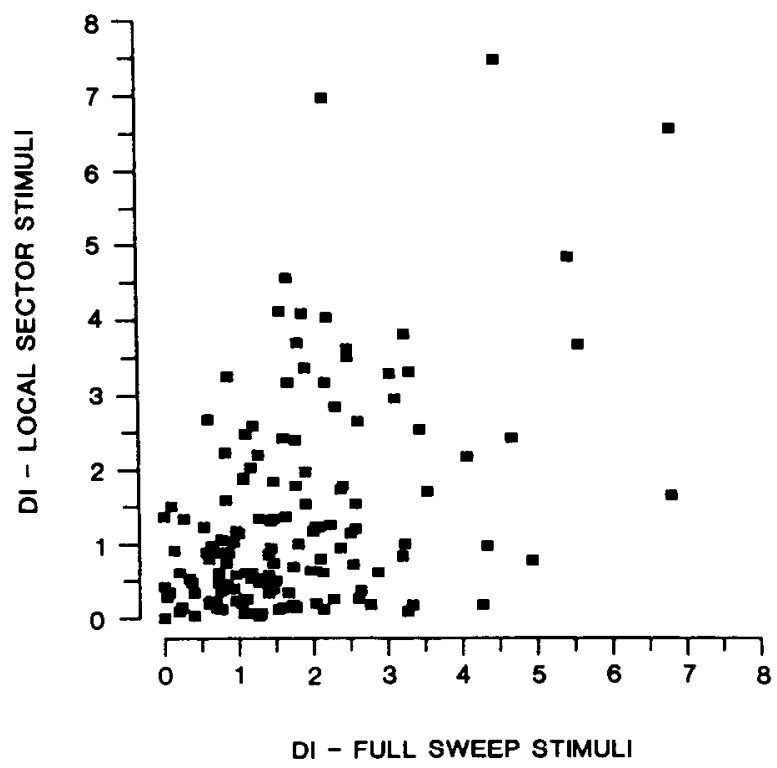

Figure 9. Comparison of the directional indices measured for full-field and local stimuli in a subset of the total number of visual field sectors tested for the population of PVNs studied. The subset consists of all $10^{\circ}$ sectors in which significant responses were evoked by both full-field and local stimuli. A generally positive relationship exists, but it is not possible to predict whether a particular sector directionally sensitive to full-field stimuli will be directionally sensitive to the local stimuli.

directional selectivity, response intensities, and receptive field sizes of PVNs are relatively independent of differences in stimulus speed over a wide range of velocities, as we show in the following section.

\section{Sensitivity of PVNs to different stimulus speeds}

The sensitivity of PVNs to stimulus speeds was tested on the meridian and in the axial direction along which stimuli evoked maximal responses in the initial field study of each PVN, like that of Figure 3, over the range of $5^{\circ}-200 \% \mathrm{sec}$. Seventy-three PVNs were studied sufficiently to allow analysis. Of these, the responses of 30 fit a linear model $(r>0.7), 16$ with positive and 14 with negative slopes of changes in discharge rate with increasing stimulus speed. The population response profiles of these PVNs are shown in Figure 14, $A$ and $B$. They show a 2-fold increase or decrease in impulse discharge frequency over the range tested, and the variation between response profiles of PVNs is large.

Other PVNs had maximal (12 of 73) or minimal (11 of 73) discharge rates evoked by stimulus speeds in the middle of the range tested. The population response profiles of these 2 groups are given in Figure 14,C and $D$. For the latter, the $V_{\max }$ or the $V_{\min }$ for each neuron was set to zero at midscale, and other stimulus speeds were normalized for each neuron as deviations in either direction. Neural response values were normalized for each neuron as the percentage of maximal response. The mean $V_{\max }$ for the population of Figure $14 \mathrm{C}$ is $122 \%$ sec (SD $=18 \%$ sec), and the mean $V_{\min }$ for the population of Figure 14 is $106 \%$ $\sec (\mathrm{SD}=13 \% \mathrm{sec})$, values near the midrange of speeds tested. This indicates that PVNs are not narrow bandpass cells as regards their sensitivity to the speed of stimuli moving in a frontoparallel plane. The remaining cells $(20$ of 73$)$ of the population 

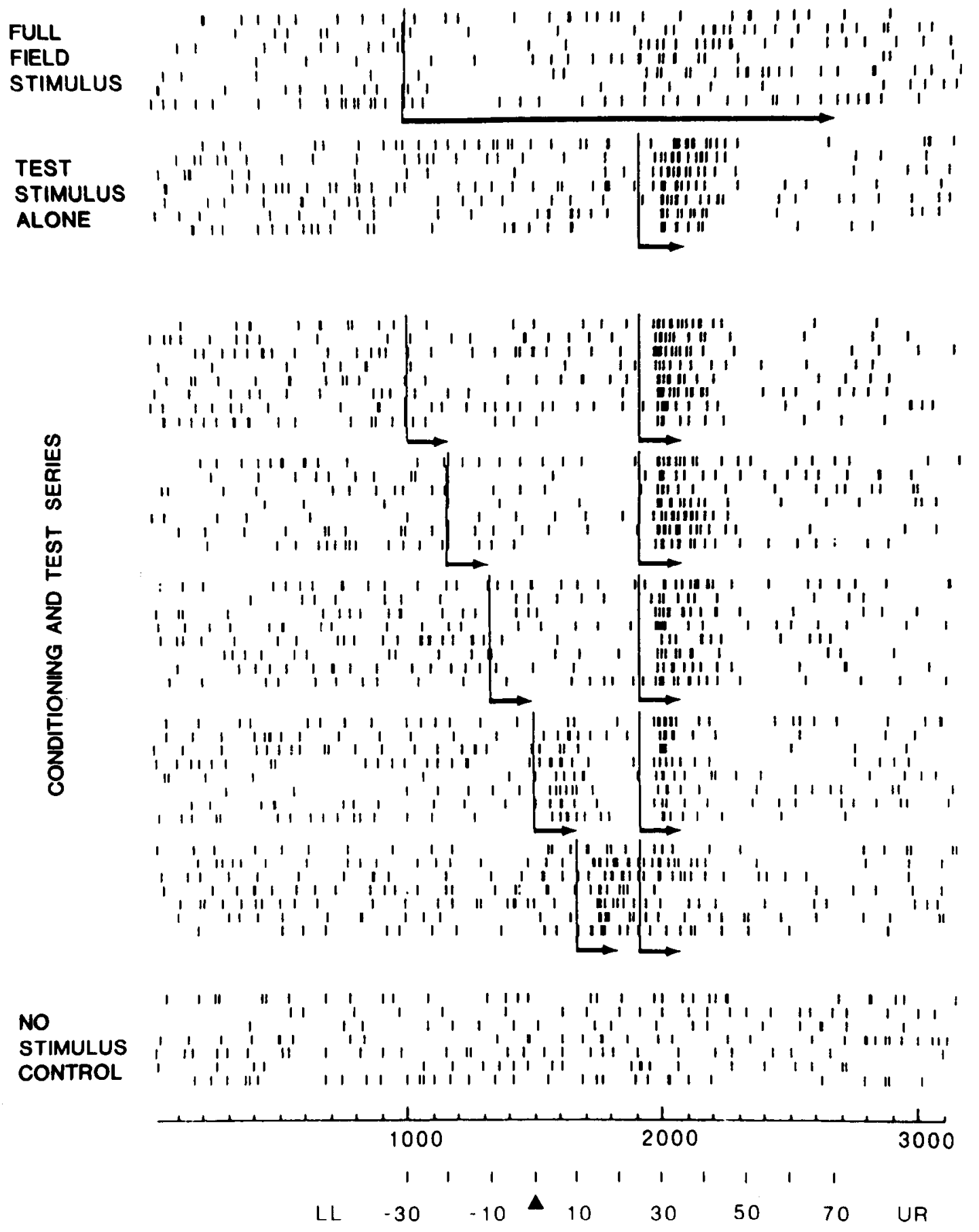

Figure 10. Illustration of the responses of a PVN in a conditioning-test paradigm used to mimic the normal temporal and spatial relations existing during a full-field stimulus. Arrows below each set of response replicates indicate the onset and duration of the local stimuli. The top 2 sets of responses contrast the responses to the local test stimuli with the lack of response to the full-field stimulus as it passed in the null direction through the same spatial location. The central 5 sets of responses show that the $10^{\circ}$ local conditioning stimuli effectively suppressed the responses to the $10^{\circ}$ local test stimuli when they were relatively close to the test location. The average rate of neuronal discharge during the interval from 50 to 200 msec after the test stimulus onset was 48 impulses/sec for the test stimulus alone and $51,49,40,31$, and 19 impulses/sec for the successive conditioning-test pairs. The bottom set of conditioning stimuli also elicited responses in a region unresponsive to the full-field stimulus. The last set of records was collected in control trials in which no stimuli were delivered. All trial sets shown in this figure were presented in a randomly intermixed fashion. Time base (in msec) translated into degrees in the visual field below. Degrees of visual angle are indicated along the diagonal from lower left $(L L)$ to upper right $(U R)$, with the point of fixation marked by the solid triangle. 
FULL

FIELD

STIMULUS

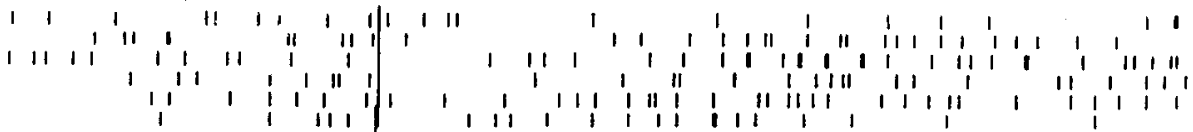

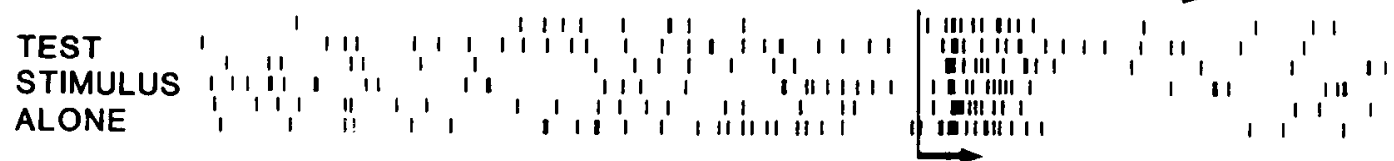

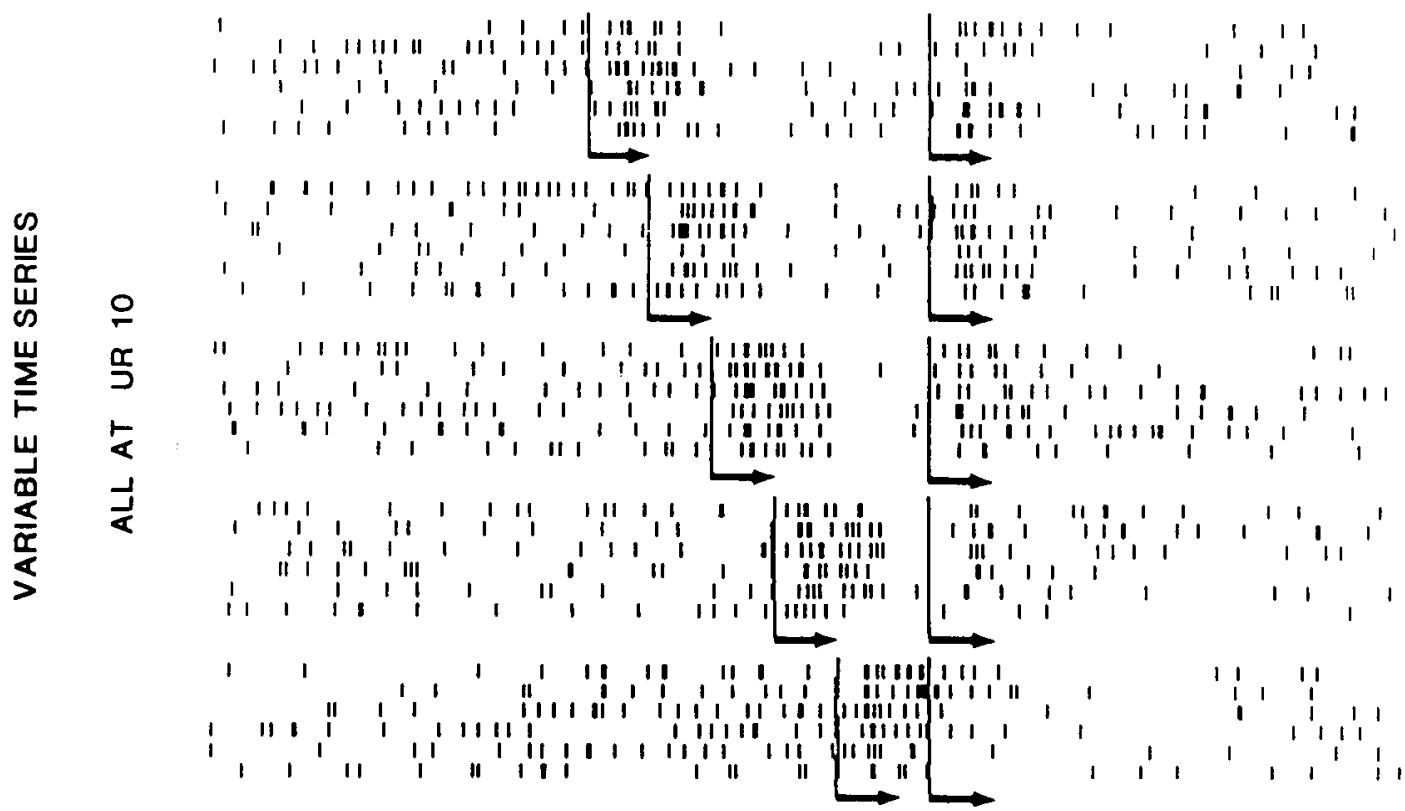

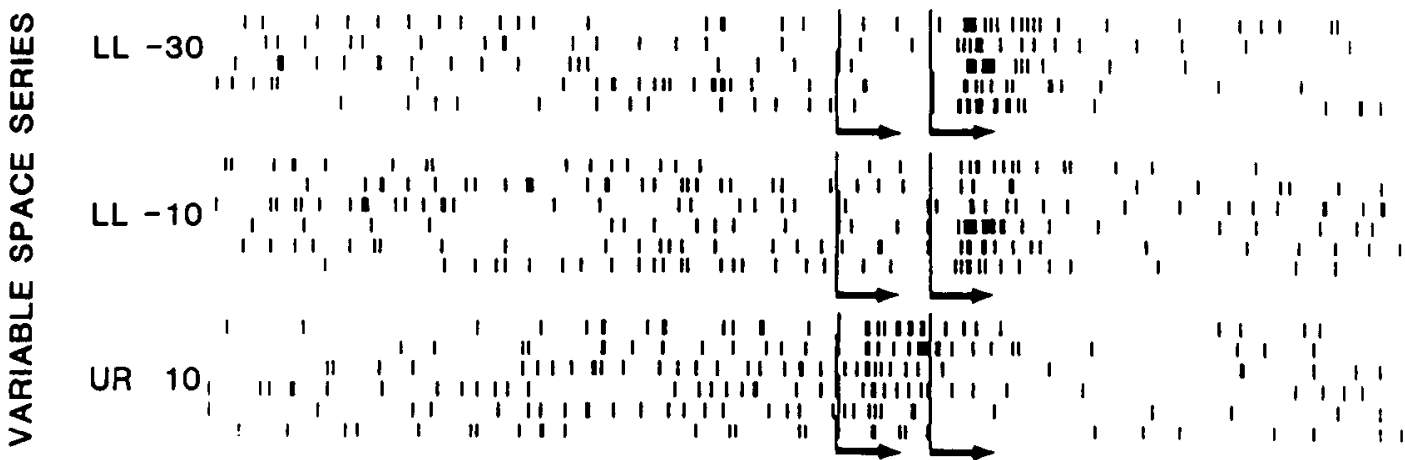

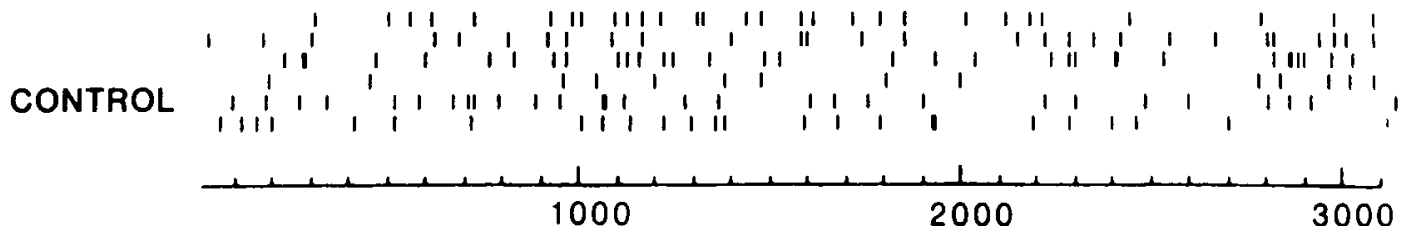

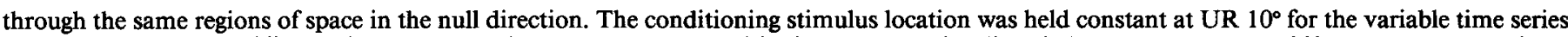

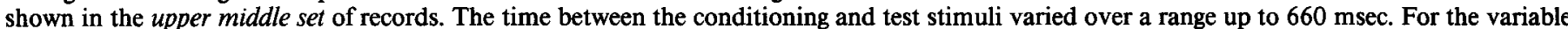

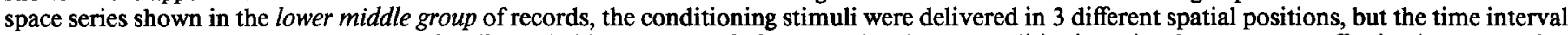

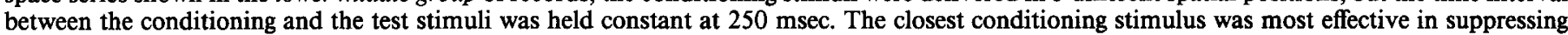

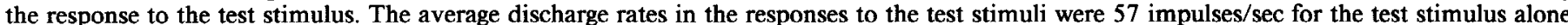
and $25,25,24,15$, and 10 impulses/sec for the time series, reading from top to bottom; and 56,42 , and 10 impulses/sec for the spatial series. 

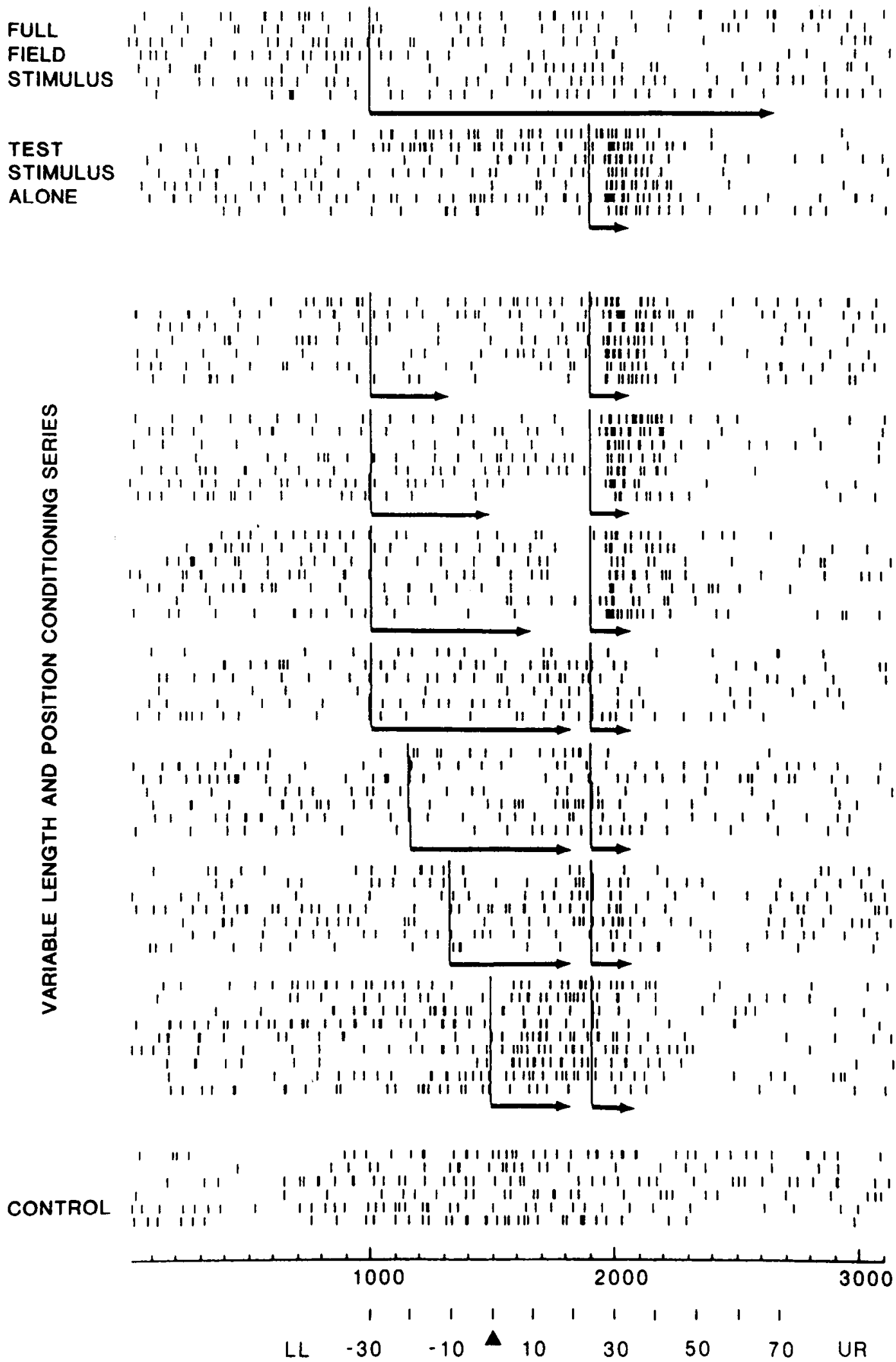

Figure 12. Results of test of the duration and spatial extent of the feed-forward inhibition thought to produce the long-range effects observed. Conventions as for Figure 10. The upper 2 sets of records contrast the full- and short-segment tests of responsiveness in the null direction. The conditioning-test series includes conditioning stimuli that traversed $20^{\circ}, 30^{\circ}, 40^{\circ}$, and $50^{\circ}$, with either the starting or the terminal position held constant. The test stimulus was always $10^{\circ}$ in spatial extent. The results illustrate that the conditioning stimulus need not elicit a response to be effective in suppressing the response to the test stimulus, that the conditioning stimulus is most effective when it invades the segment of space adjacent to that of the test stimulus, and that passage of the conditioning stimulus through more distant spatial locations also produces suppressive effects. The average rates of discharge in the responses to the test stimuli were 51 impulses/sec for the test stimulus alone and $54,47,38,14,11$, 18 , and $16 \mathrm{impulses} / \mathrm{sec}$ for the conditioning-test series, reading from top to bottom. 

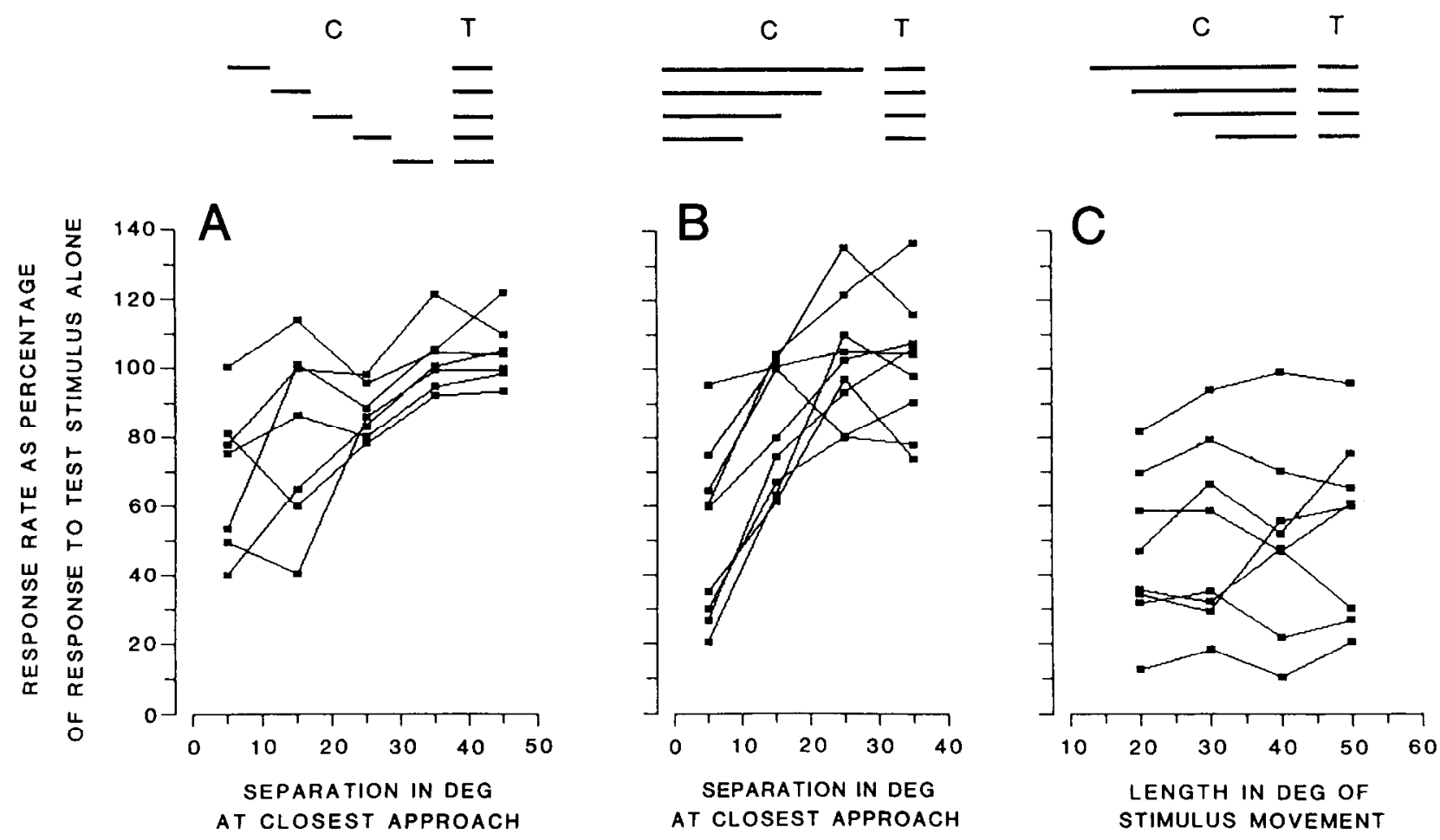

Figure 13. Line graphs illustrating the suppression of response to the test stimulus as a function of the proximity of the conditioning stimulus. Bar pictograms at the top show the spatial relations between conditioning $(C)$ and test $(T)$ stimuli for each paradigm in the graphs below. Each horizontal pair of $\mathrm{C}$ and $\mathrm{T}$ bars depicts a scaled representation of the stimulus movements (left to right) in both space and time. Stimulus speed was $60^{\circ} / \mathrm{sec}$. Each set of connected points in the graphs below gives the results for a different PVN. $A$, Conditioning stimulus movements of $10^{\circ}$ length were effective in suppressing the test response only when the movements were within $10^{\circ}-20^{\circ}$ of the test stimulus location. $B$, Suppressive effects produced by longer conditioning stimulus movements still depended upon those stimuli entering the $10^{\circ}-20^{\circ}$ area just proximal to the test stimulus area. $C$, Length of the conditioning stimulus movement prior to entering the locations just proximal to the test location had no clcar influence upon the degree of suppression.

analyzed were sensitive to stimulus motion but not to stimulus speed.

Further analyses of each of the 73 cells in these populations revealed that the spatial extent of the receptive fields varied only slightly with stimulus speed. A typical result is shown in Figure 15 for a neuron with a positive linear correlation between stimulus speed and response $(r=+0.90)$. The receptive field of the cell is large and bilateral and includes the point of fixation; the locus of peak response is at or close to that point. More extended studies of this sort along many meridians revealed a marked directional preference for stimuli moving inwardly toward the point of fixation, in the manner illustrated in Figure 3. Similar results were obtained in studies of cells of each of the classes of Figure 14.

We examined the effect of speed upon directional preference in 6 neurons. Although the intensity of the directional preference changed with changes in speed, as did response intensity, we observed no case in which the selected direction was reversed. We concluded that the directional sensitivity of PVNs, like receptive field size and location, is relatively independent of stimulus speed.

A subset of 52 of these neurons was studied for speed sensitivity when the spatial extent of stimulus movement was limited to $20^{\circ}$. A completely consonant set of results was obtained. This is, we believe, strong evidence that our observations that PVNs are relatively insensitive to differences in stimulus speed are not the result of the tangent error for speed that occurs at wide eccentricities. The error over $20^{\circ}$ of the tangent screen is small. Moreover, when 2 or more $20^{\circ}$ sectors could be tested at different positions within a neuron's receptive field along a single meridian, similar profiles of speed insensitivity were observed.

In summary, the speed of a stimulus moving along a frontoparallel plane through the visual field of a monkey engaged in an attentive fixation task is signaled imprecisely by individual PVNs. Two populations of PVNs provide reciprocally increasing and decreasing frequencies of discharge with increases in stimulus speed over the range of $30^{\circ}-200^{\circ} / \mathrm{sec}$, but the peak discharge rate changes by a factor of only about 2 over that range. The responses of neurons with positive stimulus response slopes frequently saturate for speeds above $200 \% \mathrm{sec}$ but continue to respond to those of several hundred degrees per second. Two other populations of PVNs have broadly centered maxima and minima in the middle of the range tested (at $110^{\circ}-120 \%$ sec): PVNs are not narrow bandpass cells in the sense that many neurons of the striate and prestriate areas are. Thus, the extraordinary sensitivity of PVNs to stimulus motion and to the direction of that motion is coupled with a relative insensitivity to changes in stimulus speed. The receptive field sizes and locations and the directional selectivity of PVNs are relatively unchanged with changes in stimulus speed over the ranges of response sensitivity.

\section{Discussion}

The results of the present experiments extend earlier observations of PVNs (Motter and Mountcastle, 1981) and provide new information concerning the spatial organization of their directional preferences within the visual field and the neural mechanisms thought to produce it. We discuss first how PVN recep- 

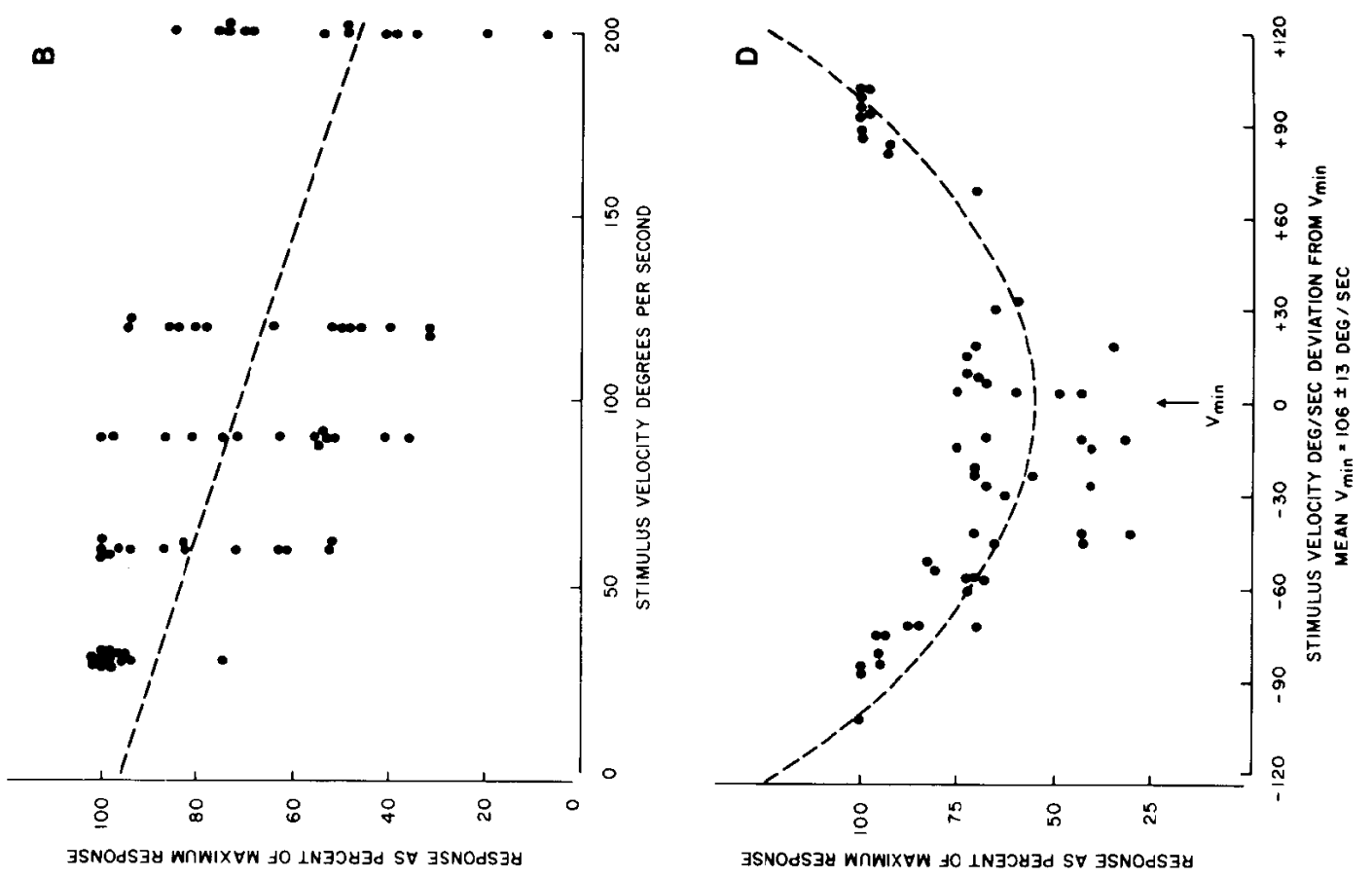

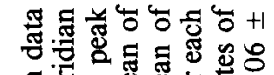

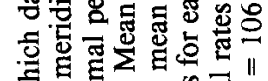

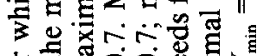

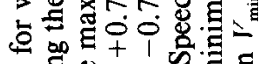

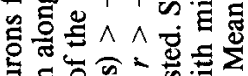

过

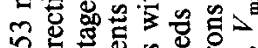

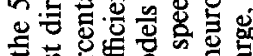

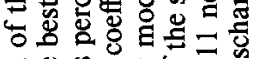

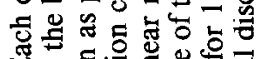

出.

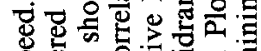

o

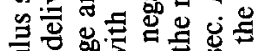

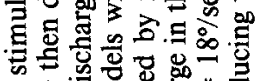

क o

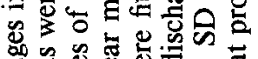

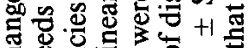

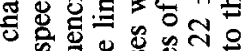

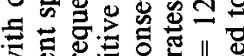

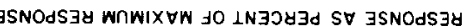

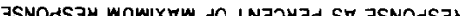

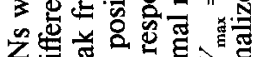

字宫㩆

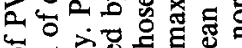
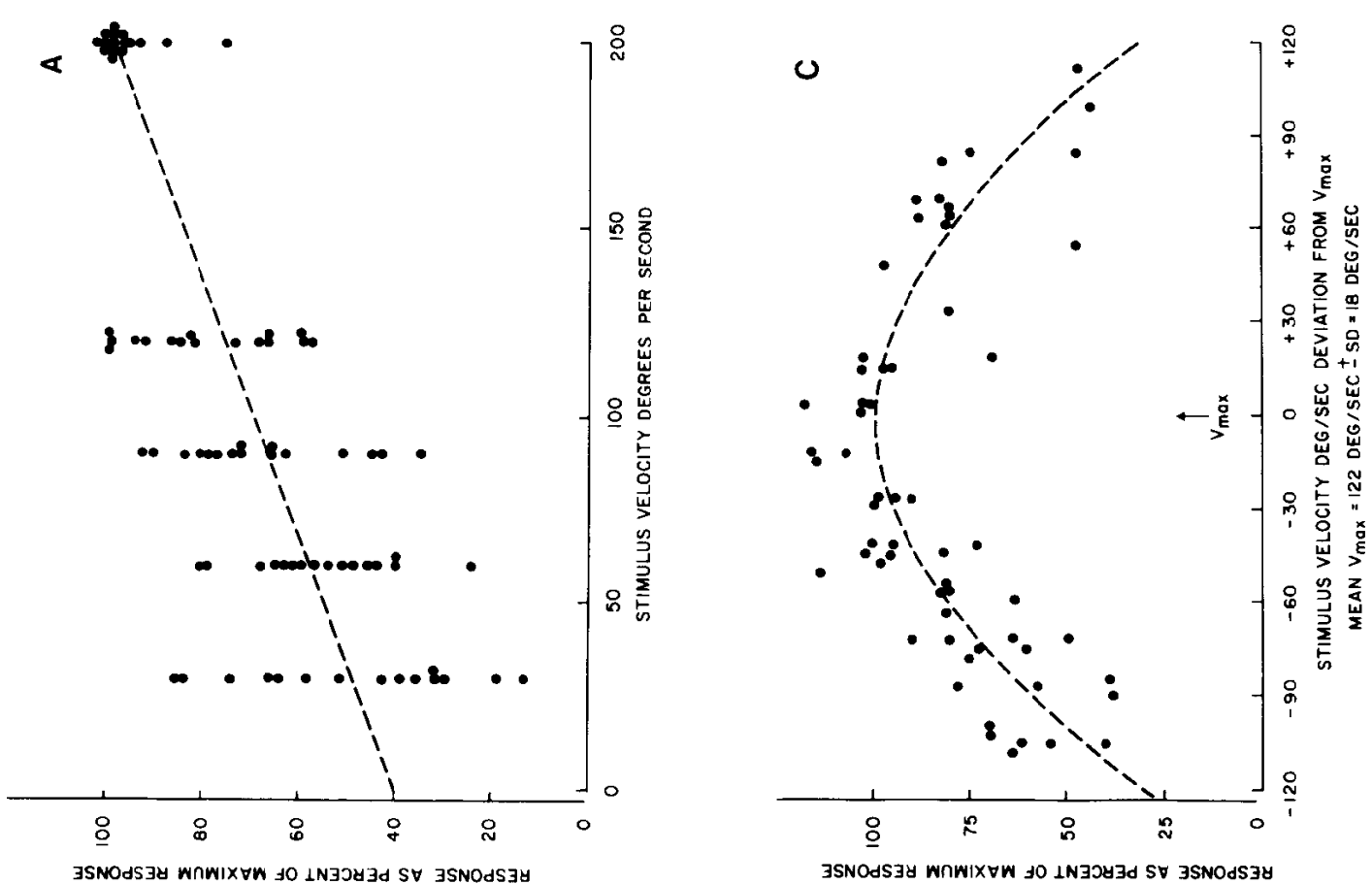

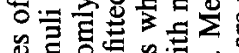

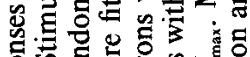

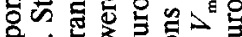

किं

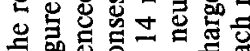

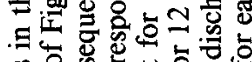

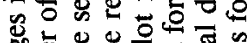

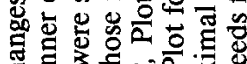

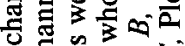

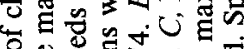

of

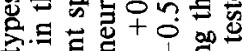

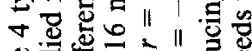

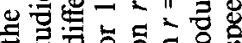

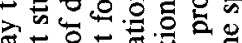

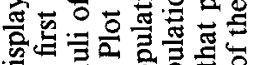

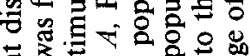

可

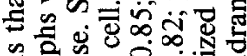

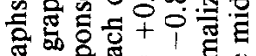

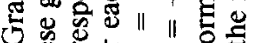

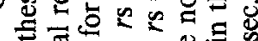

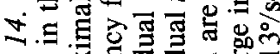

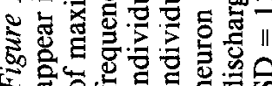


T E M P O R A L

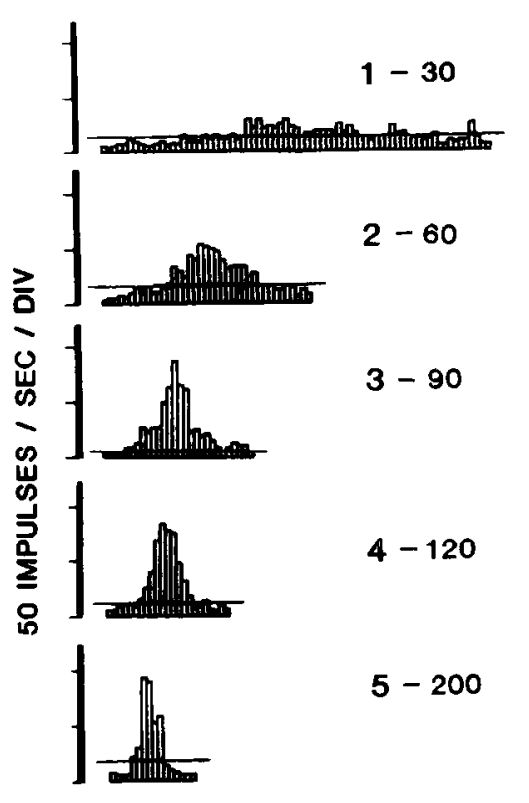

S P A T I A L
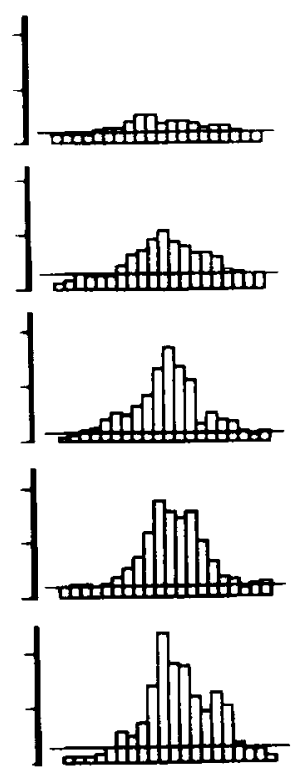
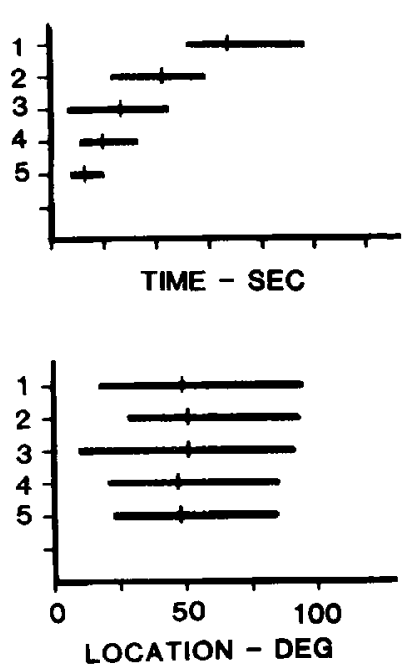

Figure 15. Results of study of the durations and spatial extents of the responses of a PVN whose peak responses changed as a positive linear function $(r=+0.90)$ of stimulus speed over classes $1-5$ at $30^{\circ}, 60^{\circ}, 90^{\circ}, 120^{\circ}$, and $20^{\circ} / \mathrm{sec}$, respectively. Identical stimuli at different speeds were delivered in randomized sequence; stimuli moved in the optimal direction, right to left, for $100^{\circ}$ along the axis meridian of best response, the horizontal meridian, centered on the point of fixation. Time histograms (left): bin size, 50 msec; horizontal line on $y$-axis is placed at $2.5 \times$ the SD of the background activity. Each histogram gives the period of stimulation, the duration of the response above the level of significance, and the time line for classes $1-5=3333,1667,1111,833$, and $500 \mathrm{msec}$, respectively. Spatial histograms (center): bin size, $5^{\circ}$; horizontal lines, significance level as above. Each histogram gives the spatial extent of the stimuli, $100^{\circ}$ for each class, and the spatial extent of the response area above the level of significance. Graph (upper right), derived from the time histograms, shows shortening of the response period (horizontal lines) and of the latency to peak response (short vertical lines) with increases in stimulus speed, classes 1-5. Graph (lower right), derived from the spatial histograms, shows that the spatial extent of the receptive field and the locations of the peak responses were almost independent of stimulus speed over the range tested.

tive fields could be produced by a projected convergence of neurons in the several transcortical systems that link the primary visual cortex to the inferior parietal lobule. We then consider whether the property of opposing directional preferences along the same meridian might be created by projected properties, by intracortical processing within $\mathrm{PG}$, or by both, and we propose a model that generates new questions for future experiments. The functional properties of the visual and other neurons of the parietal cortex suggest that the system is suited to function in the visual guidance of projected movements of the arm and in locomotion. These 2 subjects are discussed in the following paper (Steinmetz et al., 1987), together with comments on what we regard as the synthetic functions of the parietal system in the visual aspects of spatial perception. We first summarize the anatomical connections of the caudal part of the inferior parietal lobule.

\section{Anatomical considerations}

The inferior parietal lobule (IPL) of the macaque monkey contains a number of cytoarchitectural areas (Seltzer and Pandya, 1980; Pandya and Seltzer, 1982; Andersen et al., 1985; Gattass et al., 1985). Area PG occupies the caudal crown of the IPL and extends anteriorly to include the outer 3-4 $\mathrm{mm}$ of the inferior bank of the intraparietal sulcus (IPS) and posteriorly for some uncertain distance over the rim of the superior temporal sulcus (STS). PG is bordered posteromedially along the gyral axis by an area called OPT by Seltzer and Pandya (1980) that appears nearly equivalent to area PO (Covey, 1982); PG may also border a part of area $\mathrm{V} 3 \mathrm{~A}$ in this region. Anterolaterally along the same gyral axis, PG borders the transitional cortex linking PG to PF. Anteriorly, PG is bordered by area POa, which occupies the inferior bank of the IPS from its fundus to within 3-4 $\mathrm{mm}$ of the surface of the hemisphere. POa appears to contain several subfields, among them zones called VIP (Maunsell and Van Essen, 1983) and LIP (Andersen et al., 1985). Posteriorly, PG borders a number of fields that separate it from the middle temporal area (MT, or V5; Van Essen and Maunsell, 1979; Seltzer and Pandya, 1980; Newsome and Wurtz, 1982; Maunsell and Van Essen, 1983; Saito et al., 1986).

\section{Thalamic projections to the inferior parietal lobule}

The pulvinar nuclear complex is the major source of thalamocortical projections to the parietal homotypical cortex (Le Gros Clark and Northfield, 1937). This has been confirmed and extended in tracer studies in which less dense projections from a number of other thalamic nuclei were identified (Baleydier and Mauguiere, 1977; Divac et al., 1977; Kasdon and Jacobsen, 1978; Mauguiere and Baleydier, 1978; Pearson et al., 1978; Asanuma et al., 1985). Asanuma et al. (1985), for example, found that area $7 \mathrm{a}$, in their designation a subdivision of $\mathrm{PG}$, receives its major, but not its only, thalamic projection from the medial nucleus of the pulvinar, PUL-m; similarly, area $7 \mathrm{~b}$ (PF) from the PUL-o; and LIP, a subdivision of POa, from PUL-1. These subdivisions of the pulvinar receive different afferent inputs: PUL-m from the deep but not from the retinorecipient upper layers of the superior colliculus (SC) (Benevento and Fallon, 1975; Benevento et al., 1977; Harting et al., 1980; Benevento and Standage, 1983); PUL-1, by contrast, receives 
descending projections from the prestriate cortex and ascending ones from retinorecipient $\mathrm{SC}$. Thus, POa appears to receive visual afferent input from a number of indirect pathways, including the transcortical one described below, whereas PG depends mainly upon the latter for input of visual origin.

\section{Multistage transcortical projection from the striate cortex to the inferior parietal lobule}

It is now established that the striate cortex projects forward to engage, both directly and over interrupted relays, a number of areas in the prestriate visual cortex. These areas differ in their patterns of corticocortical connections and in the functional properties of their neurons (for reviews, see Zeki, 1978; Van Essen, 1979; Van Essen and Maunsell, 1979; Ungerleider, 1985). Two divergent but partially interlocking outputs from these systems project upon the neocortex of the temporal and parietal lobes. These anatomical findings and the results of lesion studies in man and other primates are the basis for the idea that these 2 divergent transcortical pathways serve different aspects of perception: that to the temporal lobc, object recognition, particularly form and color; and that to the parietal lobe, the spatial relations between objects and more global aspects of spatial perception (Gross, 1973; Mishkin and Ungerleider, 1982; Ungerleider and Mishkin, 1982; Mishkin et al., 1983). Lesions of the IPL produce disorders of spatial perception, and it is this region, particularly $P G$, that is a principal target of the striatoparietal system.

Area $P G$ receives no direct input from the striate cortex. Its ipsilateral inputs conveying signals of visual origin are from immediately adjacent areas: (1) POa (Mesulam et al., 1977; Seltzer and Pandya, 1980), (2) PO (Pandya and Seltzer, 1982), and (3) the cortex of the anterior bank and floor of the STS, including the middle superior temporal area, MST (Mesulam et al., 1977; Lane, 1982; Seltzer and Pandya, 1984). These 3 regions are, in turn, targets of relayed projections that originate in the striate cortex.

1. POa receives a strong projection from all parts of the V5 (MT) (Van Essen and Maunsell, 1979: Ungerleider et al., 1980; Rockland and Pandya, 1981; Maunsell and Van Essen, 1983; Ungerleider, 1985); and V5 (MT) receives convergent projections from all parts of the striate cortex and from intervening prestriate areas V2, V3, and VP (Zeki, 1971, 1978; Montero, 1980; Ungerleider and Mishkin, 1982; Burkhalter and Van Essen, 1983; Maunsell and Van Essen, 1983; Ungerleider et al., 1983; Weller and Kaas, 1983; DeYoe and Van Essen, 1984; Felleman and Van Essen, 1984; Felleman et al., 1984). The cells of the upper layers of V2 that project to V4 and V5 (MT) are segregated into separate cytochrome oxidase defined regions (stripes) of V2. Those neurons projecting to V5 (MT) are thought to lie in the densely staining stripes and are themselves, like the neurons of the V5 (MT), strongly selective for stimulus motion and direction, less so for orientation, and rarely for the wavelength of light (DeYoe and Van Essen, 1985; Shipp and Zeki, 1985).

2. PO receives projections from V1, V2, V3, and VP, particularly from their representations of the periphery of the visual field (Seltzer and Pandya, 1980; Covey, 1982; Colby et al., 1983; Ungerleider, 1985).

3. MST and adjacent fields within the STS received projections from V5 (MT) (Seltzer and Pandya, 1978; Maunsell and Van Essen, 1983; Ungerleider, 1985) and from anterior V4 (Felleman and Van Essen, 1983). The PG is densely and recip- rocally connected with its homolog of the opposite hemisphere (Lane, 1982; Asanuma et al., 1985) and with the cortex of the principal sulcus of the frontal lobe. It projects to 3 separate areas in the superior temporal sulcus and to 3 different areas on the ventral surface of the temporal lobe, the parahippocampal gyrus, the presubiculum, and the perirhinal cortex (Seltzer and Pandya, 1984).

\section{Mechanisms generating the receptive fields of parietal visual neurons}

The receptive fields (RFs) of neurons in cortical areas of the transcortial projection system increase in size from V1 through V5. The RFs of V2 and V3 are observed to be "larger than those of V1" (Baizer, 1982); those of V4 are 1-2 orders larger than those of V1, varying with eccentricity (Desimone et al., 1985); and those of V5 range from 100 to $400 \mathrm{deg}^{2}$ in area (Dubner and Zeki, 1971; Zeki, 1974; Gattass and Gross, 1981; Newsome et al., 1983; Felleman and Kaas, 1984; Tanaka et al., 1984). These fields are even larger in the areas penultimate to PG, areas MST, PO, and POa. Thus, it is likely that the giant RFs of PVNs are produced by convergent input from these sources. Some of the RFs of V5 extend for a few degrees across the vertical meridian (Dubner and Zeki, 1971), and we have observed a similar spread for RFs of neurons of V4 (V. B. Mountcastle, B. C. Motter, and M. A. Steinmetz, unpublished observations). However, PG appears to be the first area in this pathway in which many neurons subtend giant bilateral RFs that may extend into the far periphery of both visual half-fields, including the monocular rims. It appears likely that this bilaterality depends upon the dense connections between the areas PG of the 2 hemispheres via the corpus callosum (Lane, 1982). The presence of a few small, a few purely ipsilateral, and some purely contralateral PVN RFs (Fig. $5 F$ ) indicates that both ipsilateral and contralateral projected inputs to $P G$ are further converged and summed within the neuronal processing chains of this area. Some of the ncurons of the polysensory area of the superior temporal sulcus (PST) subtend RFs that resemble the giant fields of PVNs in size, bilaterality, and directional properties (Bruce et al., 1981). Whether the RFs of PST neurons are generated independently or represent projected properties of PVNs is unknown, but the heavy projections from PG to PST (Ungerleider et al., 1983) suggest that this may be the case.

The receptive fields of PVNs vary in size with the state of attention (Mountcastle et al., 1983) and the angle of gaze (Andersen and Mountcastle, 1983). They also differ with differences in the parameters of the stimuli used to establish them. The giant size and the variability of the RFs of single PVNs bring into question the usefulness of the receptive field concept as a denominating property of cortical neurons, at least at this level of the homotypical cortex and at this degree of complexity of central neural synthetic mechanisms.

\section{Mechanisms generating the directionalities of PVNs}

The opponent directional patterns of PVNs require for their composition anisotropic effects organized in the centrifugal and centripetal directions for the inward and outward classes of neurons, respectively. This is suggested by the analyses of directionality along a single axis described above and the broad sinusoidal distributions of axial selectivities in the circular dimension analyzed in the following paper (Steinmetz et al., 1987). It is unlikely, we believe, that such a pattern could be composed 


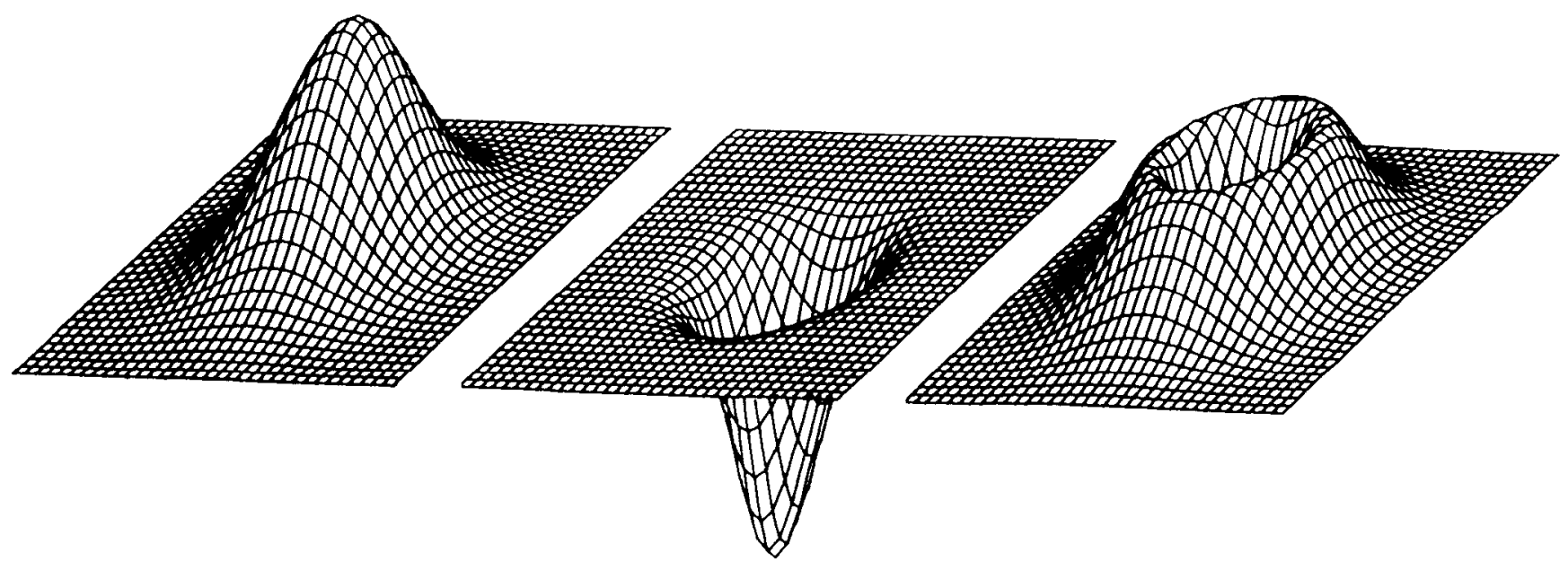

Figure 16. Three-dimensional, double-Gaussian model representing the responses of a PVN sensitive to inward stimulus motion. The horizontal and vertical dimensions of the visual field are represented on the $x$-and $y$-axes, respectively, and the response amplitude on the $z$-axis. Left, An excitatory Gaussian with parameters determined by the convergence of extrinsic excitatory inputs to the cell. Center, An inhibitory Gaussian with a narrower spatial distribution than the excitatory one; it is thought to be generated by dynamic intrinsic cortical mechanisms. In this example, both excitatory and inhibitory Gaussians are centered on the point of fixation. Right, Arithmetic sum of the distributions of the excitatory and inhibitory Gaussians. It results in a response surface similar to ones often observed for PVNs, e.g., the neuron of Figures 3 and 4 . Variation in the intensity of the inhibitory component is thought to determine whether the receptive field includes or spares the foveal region. A reciprocal model explains in a similar way the receptive fields and directional properties of neurons with outward directionality, like that of Figure $5 B$.

by a convergent summation of the directional properties of cells that project upon PG: first, because we observed no single case for which the full-field organization of directionalities for a PVN could be explained by differences in local axial selectivity in different parts of the PVN receptive field (see Fig. 3, following paper); second, because the opponent organization along several meridians distributed in equal intervals in the circular dimension would require an incredible specificity of projected convergences, including interhemispheric ones, to compose the directional patterns of PVNs with receptive fields such as those of Figures 3 and 4, $A-E$.

The results of our conditioning-test experiments (Figs. 1012) suggest that the required anisotropic effect is a feed-forward inhibition operating in the null direction and generated locally within the parietal cortex and that this effect extends for tens of degrees across the field in the null direction in front of a moving stimulus. This accords with the classic model for visual neuronal directional preferences of Barlow and colleagues derived from their studies of the rabbit retina (Barlow et al., 1964; Barlow and Levick, 1965); that is, that the receptive field of a visual neuron consists of a series of subunits each not itself directionally selective. Directionality results because each subunit when activated inhibits its neighbors on one side and not the other, yielding the familiar pattern of "preferred" and "nonpreferred" directions of stimulus movement.

There is now strong evidence that feed-forward inhibition extended in the null direction produces the directional selectivity (DS) of neurons in cat V1 and V2 (Pettigrew et al., 1968; Goodwin and Henry, 1975; Goodwin et al., 1975; Emerson and Gerstein, 1977a, b; Bishop et al., 1980; Orban et al., 1981). Directional responses are evoked by stimuli that move as little as $1 \mathrm{~min}$ of arc and disappear if that movement occurs across a narrow window. The RFs of V1 DS neurons consist of subunits between which there is nonlinear summation and an asymmetric distribution of inhibition (Ganz and Felder, 1984), which has been confirmed by intracellular recording (Creutzfeldt et al.,
1974). Intracortical infusion of the GABA inhibitor bicuculline eliminates DS for some but not all visual cortical DS neurons (Sillito, 1977). Cats reared in stroboscopic lighting show a reduced proportion of DS neurons in V1 (Kennedy and Orban, 1983). Sequentially placed stationary stimuli within the RF, delivered at optimal time intervals, elicit DS responses like those evoked by moving stimuli (Emerson and Coleman, 1979; Cremieux et al., 1984) On the basis of findings such as thesc, the Barlow-Levick model for the generation of directionality can be regarded as established for neurons of the primary visual area of the cat.

The evidence for this model of directional mechanisms in visual cortical areas in the monkey is best for area V5 (MT). Newsome et al. (1982) and Mikami et al. (1983) demonstrated an inhibition in the null direction for MT neurons using the sequentially placed stationary stimulus paradigm, as has $\mathrm{Al}-$ bright (1984), who extended the analysis to 2 dimensions to account for his observations of MT cells.

The most unusual feature of the directionality of PVNs is the great extension in space and duration of the feed-forward inhibition observed (see Figs. 11, 12): It extends forward for $15^{\circ}-$ $20^{\circ}$ ahead of a moving stimulus, and it persists for hundreds of milliseconds. Moreover, the direction of the inhibition appears to change gradually from meridian to meridian in the visual field and to be oriented along all meridians in either the centripetal or centrifugal directions. Our observations are still insufficient to establish whether the directional properties of PVNs can be understood by a direct application of the Barlow-Levick model, for we have not yet studied opponent organization in the conditioning-test paradigms of Figures 10-12. In addition, we have preliminary evidence that inhibition may also be extended in the preferred direction, but this inhibition has not been studied quantitatively.

There is a model that accounts for our observations on opponent organization. Assume that each PVN is related to large, superimposed, and almost completely overlapping inhibitory 
and excitatory RFs, within which there is minimal local directionality. At the limit, these fields cover the entire visual field but have different spatial profiles of response intensity, illustratcd by the double-Gaussian model in Figure 16 (Rodieck and Stone, 1965). It is well known that inhibitory synaptic events in cortical neurons are of much greater duration than are excitatory ones (Stephanis and Jasper, 1964; Creutzfeldt et al., 1966). Then, with reciprocal models for inward and outward opponent organization of the directionalities of PVNs, our observations can be accounted for as follows (for the inward case).

1. The strong inward directionality, arranged in an opponent fashion along single meridians, is produced by the long-range, feed-forward inhibition operating at different intensities in the 2 directions. This model is quite similar to that used by Heggelund $(1981 \mathrm{a}, \mathrm{b})$ in his studies of the receptive field organization of simple and complex cells in the cat striate cortex. The inhibition increments as stimuli move inwardly from the periphery towards the center of gaze, quenching the response to inward movement and preventing any response on the outward half of the axial movement. The size of the central zone of sparing and the question of whether it exists at all are accounted for by differences in the intensity profiles of the excitatory and inhibitory receptive fields for different PVNs.

2. The absence of a sharp axial selectivity and the centripetal directionality along all meridians are accounted for by the extension of the double-Gaussian model in 2 dimensions, where it is usually asymmetric. A smooth, sinuosoidally distributed variation in response intensity along different axes consecutively in the circular dimension is described in the following paper (Steinmetz et al., 1987).

3. The decrease in the incidence and intensity of directional responses with decreases in the spatial length of moving stimuli, i.e., with decreases in the intensity of the inhibitory effect, is accounted for by the greater spatial and temporal summation of inhibition evoked by stimuli of greater spatial extent.

4. The nondirectional responses to short, moving stimuli within the central zone of sparing of response to full-field stimuli that are frequently observed (Fig. 6) are accounted for by the rapid onset of excitation and more slowly accumulating inhibition evoked by these stimuli.

This model accounts for the facts presently available. But whether it, the classic Barlow-Levick model, or some other will prove most adequate is still uncertain. The double-Gaussian model places a minimal demand upon the specificity of projected properties and upon intracortical processing mechanisms. It requires a wide spatial convergence of excitation and inhibition upon PVNs and a differential distribution of the intensity profiles of the 2 receptive fields.

Organizations of directionalities similar to that of PVNs have been described in the cat by Orban et al. (1981). DS neurons of area 18 preferred centrifugal directions, while some area 17 DS cells preferred centrifugal and others centripetal directions of stimulus movement. Finally, Bruce et al. (1981) described some cells of the polysensory area of the temporal lobe (PST) with directional organizations similar to those of PVNs. It is unknown whether the directional organization for cells of PST depends uniquely upon the heavy projections from PG to PST (Seltzer and Pandya, 1984) or is generated independently.

\section{References}

Albright, T. D. (1984) Direction and orientation selectivity of neurons in visual area MT of the macaque. J. Neurophysiol. 52: 1106-1130.
Andersen, R. A., and V. B. Mountcastle (1983) The influence of the angle of gaze upon the excitability of the light sensitive neurons of the posterior parietal cortex. J. Neurosci. 3: 532-548.

Andersen, R. A., G. K. Esseck, and R. A. Siegel (1984) The role of eye position in the visual response of neurons in area 7A. Soc. Neurosci. Abstr. 10: 934.

Andersen, R. A., C. Asanuma, and W. M. Cowan (1985) Callosal and prefrontal associational projecting cell populations in area $7 \mathrm{~A}$ of the macaque monkey: A study using retrogradely transported fluorescent dyes. J. Comp. Neurol. 232: 443-455.

Asanuma, C., R. A. Andersen, and W. M. Cowan (1985) The thalamic relations of the caudal inferior parietal lobule and the lateral prefrontal cortex in monkeys: Divergent cortical projections from cell clusters in the medial pulvinar nucleus. J. Comp. Neurol. 241: 357-381.

Baizer, J. S. (1982) Receptive field properties of V3 neurons in monkey. Invest. Ophthalmol. Vis. Sci. 23: 87-95.

Baleydier, C., and F. Mauguiere (1977) Pulvinar-latero-posterior afferents to cortical area 7 in monkeys demonstrated by horseradish peroxidase tracing technique. Exp. Brain Res. 27: 501-507.

Barlow, H. B., and W. R. Levick (1965) The mechanism of directionally selective units in the rabbit's retina. J. Physiol. (Lond.) 178: 477-504.

Barlow, H. B., R. M. Hill, and W. R. Levik (1964) Retinal ganglion cells responding selectively to direction and speed of image motion in rabbit. J. Physiol. (Lond.) 173: 377-407.

Benevento, L. A., and J. H. Fallon (1975) The ascending projections of the superior colliculus in the rhesus monkey (Macaca mulatta). J. Comp. Neurol. 160: 339-362.

Benevento, L. A., and G. P. Standage (1983) The organization and projection of the retinorecipient and nonretinorecipient nuclei of the pretectal complex and layers of the superior colliculus to the lateral and medial pulvinar in the macaque monkey. J. Comp. Neurol. 217: $307-336$.

Benevento, L. A., M. Rezak, and R. Santos-Anderson (1977) An autoradiographic study of the projections of the pretectum in the rhesus monkey (Macaca mulatta): Evidence for sensorimotor links to the thalamus and oculomotor nuclei. Brain Res. 127: 197-218.

Bishop, P. O., H. Kato, and G. A. Orban (1980) Direction-selective cells in complex family in cat striate cortex. J. Neurophysiol. 43: 1266-1283.

Bruce, C., R. Desimone, and C. G. Gross (1981) Visual properties of neurons in a polysensory area in superior temporal sulcus of the macaque. J. Neurophysiol. 46: 369-384.

Burkhalter, A., and D. C. Van Essen (1983) The connections of the ventral posterior area (VP) in the macaque monkey. Soc. Neurosci. Abstr. 9: 153.

Colby, C. L., R. Gattass, C. R. Olson, and C. G. Gross (1983) Cortical afferents to area PO in the macaque. Soc. Neurosci. Abstr. 9: 152.

Covey, E. (1982) A new visual area in the parieto-occipital sulcus of the macaque. Soc. Neurosci. Abstr. 8: 691 .

Cremieux, J., G. A. Orban, and J. Duysens (1984) Responses of cat visual cortical cells to continuously and stroboscopically illuminated moving light slits compared. Vision Res. 24: 449-457.

Creutzfeldt, O. D., S. Watanabe, and H. D. Lux (1966) Relations between EEG phenomena and potentials of single cortical cells (parts I and II). Electroencephalogr. Clin. Neurophysiol. 20: 19-37.

Creutzfeldt, O. D., U. Kuhnt, and L. A. Benevento (1974) An intraccllular analysis of visual cortical ncuroncs to moving stimuli: Responses in a co-operative neuronal network. Exp. Brain Res. 21: 251274.

Desimone, R., S. J. Shein, J. Moran, and L. A. Ungerleider (1985) Contour, color and shape analysis beyond striate cortex. Vision Res. 25: 441-452.

DeYoe, E. A., and D. C. Van Essen (1984) Neurons projecting to MT and V4 from macaque V2 are segregated into discrete stripe-like patches. Soc. Neurosci. Abstr. 10:934.

DeYoe, E. A., and D. C. Van Essen (1985) Segregation of efferent connections and receptive field properties in visual area V2 of the macaque. Nature 317: 58-61.

Divac, I., J. H. LaVail, P. Rakic, and K. R. Winston (1977) Heterogeneous afferents to the inferior parietal lobule of the rhesus monkey revealed by the retrograde transport method. Brain Res. 123: 197207.

Dubner, R., and S. M. Zeki (1971) Response properties and receptive fields of cells in an anatomically defined region of the superior temporal sulcus in the monkey. Brain Res. 35: 528-532. 
Emerson, R. C., and L. Coleman (1979) The special nature of movement for neurons in the cat's visual cortex. Invest. Ophthalmol. Vis. Sci. [Suppl.] p. 134.

Emerson, R. C., and G. L. Gerstein (1977a) Simple striate neurons in the cat. I. Comparison of responses to moving and stationary stimuli. J. Neurophysiol. 40: 119-135.

Emerson, R. C., and G. L. Gerstein (1977b) Simple striate neurons in the cat. II. Mechanisms underlying directional asymmetry and directional selectivity. J. Neurophysiol. 40: 136-155.

Felleman, D. J., and J. H. Kaas (1984) Receptive-field properties of neurons in middle temporal area (MT) of owl monkeys. J. Neurophysiol. 52: 488-512.

Felleman, D. J., and D. C. Van Essen (1983) The connections of area V4 of macaque monkey extrastriate cortex. Soc. Neurosci. Abstr. 9: 153.

Felleman, D. J., and D. C. Van Essen (1984) Cortical connections of area $\mathrm{V} 3$ in macaque extrastriate cortex. Soc. Neurosci. Abstr. 10:933.

Felleman, D. J., D. J. Carman, and D. C. Van Essen (1984) Evidence for a functional distinction between areas V3 and VP of macaque extrastriate cortex. Invest. Ophthalmol. Vis. Sci. [Suppl.] 25: 278.

Ganz, L., and R. Felder (1984) Mechanism of directional selectivity in simple neurons of the cat's visual cortex analyzed with stationary flash sequences. J. Neurophysiol. 51: 294-324.

Gattass, R., and C. G. Gross (1981) Visual topography of striate projection zone (MT) in posterior superior temporal sulcus of the macaque. J. Neurophysiol. 46: 621-628.

Gattass, R. A., P. B. Sousa, and E. Covey (1985) Multiple visual areas in extrastriate cortex. In Pattern Recognition Mechanisms, C. Chagas, ed., pp. 1-20, Pontifical Academy of Sciences, Vatican City.

Goodwin, A. W., and G. H. Henry (1975) Direction selectivity of complex cells in a comparison with simple cells. J. Neurophysiol. 38: $1524-1540$.

Goodwin, A. W., G. H. Henry, and P. O. Bishop (1975) Direction selectivity of simple striate cells: Properties and mechanisms. J. Neurophysiol. 38: 1500-1523.

Gross, C. G. (1973) Visual functions of inferotemporal cortex. In Handbook of Sensory Physiology, Vol. 7, R. Jung, ed., pp. 451-482, Springer, Berlin.

Harting, J. K., M. F. Huerta, A. J. Frankfurter, N. L. Strominger, and G. J. Royce (1980) Ascending pathways from the monkey superior colliculus: An autoradiographic analysis. J. Comp. Neurol. 192: 853882.

Heggelund, P. (1981a) Receptive field organization of simple cells in cat striate cortex. Exp. Brain Res. 42: 89-98.

Heggelund, P. (1981b) Receptive field organization of complex cells in cat striate cortex. Exp. Brain Res. 42: 99-107.

Kasdon, D. L., and S. Jacobsen (1978) The thalamic afferents to the inferior parietal lobule of the rhesus monkey. J. Comp. Neurol. 177: 685-706.

Kennedy, H., and G. A. Orban (1983) Response properties of visual cortical neurons in cats reared in stroboscopic illumination. J. Neurophysiol. 49: 686-704.

Lane, J. K. (1982) The modular efferent organization of the inferior parietal lobule and caudal principal sulcus for their callosal and reciprocal association projections: Computer-assisted mapping of the tangential and radial distributions of horseradish peroxidase/tetramethylbenzidine (HRP/TMB)-labelled neurons in homotypical neocortex of the rhesus monkey. Ph.D. thesis, The Johns Hopkins University, Baltimore, MD.

Le Gros Clark, W. E., and D. C. Northfield (1937) The cortical projection of the pulvinar in the macaque monkey. Brain 60: 126-142.

Lynch, J. C., V. B. Mountcastle, W. H. Talbot, and T. C. T. Yin (1977) Parictal lobe mechanisms for directed visual attention. J. Neurophysiol. 40: 362-389.

Mauguiere, F., and C. Baleydier (1978) Topographical organization of medial pulvinar neurons sending fibres to Brodmann's areas 7, 21, and 22 in the monkey. Exp. Brain Res. 31: 605-607.

Maunsell, J. H. R., and D. C. Van Essen (1983) The connections of the middle temporal visual area (MT) and their relationship to a cortical hierarchy in the macaque monkey. J. Neurosci. 3: 2563-2586.

Mesulam, M. M. G. W. Van Hoesen, D. C. Pandya and N. Geschwind (1977) Limbic and sensory connections of the inferior parietal lobule (area PG) in the rhesus monkey: A study with a new method for horseradish peroxidase histochemistry. Brain Res. 136: 393-141.

Mikami, A., W. T. Newsome, and R. H. Wurtz (1983) Mechanisms of direction and speed selectivity in the middle temporal visual area
(MT) of the macaque monkey. Invest. Ophthalmol. Vis. Sci. [Suppl.] 24: 107.

Mishkin, M., and L. G. Ungerleider (1982) Contribution of striate inputs to the visuospatial functions of parieto-preoccipital cortex in monkeys. Behav. Brain Res. 6: 57-77.

Mishkin, M., L. G. Ungerleider, and K. A. Macko (1983) Object vision and spatial vision: Two cortical pathways. Trends Neurosci. $6: 414-$ 417.

Montero, V. (1980) Patterns of connections from the striate cortex to cortical visual areas in superior temporal sulcus of macaque and middle temporal gyrus of owl monkey. J. Comp. Neurol. 189: 4559.

Motter, B. C., and V. B. Mountcastle (1981) The functional properties of the light-sensitive neurons of the posterior parietal cortex studied in waking monkeys: Foveal sparing and opponent vector organization. J. Neurosci. 1: 3-26.

Mountcastle, V. B., R. A. Andersen, and B. C. Motter (1981) The influence of attentive fixation upon the excitability of the light-sensitive neurons of the posterior parietal cortex. J. Neurosci. 1 : 12181235.

Mountcastle, V. B., B. C. Motter, M. A. Steinmetz, and C. Duffy (1984) Looking and seeing: The visual functions of the parietal lobe. In Dynamic Aspects of Neocortical Function, G. M. Edelman, W. M. Cowan, and W. E. Gall, eds., pp. 159-194, Wiley, New York.

Mountcastle, V. B., J. C. Lynch, A. Georgopoulos, H. Sakata, and C. Acuna (1975) Posterior parietal association cortex of the monkey: Command functions for operations within extrapersonal space. J. Neurophysiol. 38: 871-908.

Newsome, W. T., and R. H. Wurtz (1982) Identification of architectonic zones containing visual tracking cells in superior temporal sulcus (STS) of macaque monkeys. Invest. Opthalmol. Vis. Sci. [Suppl.] 22. 238.

Newsome, W. T., A. Mikami, and R. H. Wurtz (1982) Direction selective responses to sequentially flashed stimuli in extrastriate area MT in the awake macaque monkey. Soc. Neurosci. Abstr. 8: 812.

Newsome, W. T., M. S. Gizzi, and J. A. Movshon (1983) Spatial and temporal properties of neurons in macaque MT. Invest. Opthalmol. Vis. Sci. [Suppl.] 24: 107.

Orban, G. A., H. Kennedy, and H. Maes (1981) Response to movement of neurons in areas 17 and 18 of the cat: Direction selectivity. J. Neurophysiol. 45: 1059-1073.

Pandya, D. N., and B. Seltzer (1982) Intrinsic connections and architectonics of the posterior parietal cortex in the rhesus monkey. J. Comp. Neurol. 204: 196-210.

Pearson, R. C. A., P. Brodal, and T. P.S. Powell (1978) The projection of the thalamus upon the parietal lobe in the monkey. Brain Res. 144: 143-148.

Pettigrew, J. D., T. Nikara, and P. O. Bishop (1968) Responses to moving slits by single units in cat striate cortex. Exp. Brain Res. 6 : 373-380.

Rockland, K. S., and D. N. Pandya (1981) Cortical connections of the occipital lobe in the rhesus monkey: Interconnections between areas 17,18 , and 19 and the superior temporal sulcus. Brain Res. 212:249270.

Rodieck, R. W., and J. Stone (1965) Analysis of receptive fields of cat retinal ganglion cells. J. Neurophysiol. 28: 833-849.

Saito, H., M. Yukie, K. Tanaka, K. Hikosaka, Y. Fukada, and E. Iwai (1986) Integration of direction signals of image motion in the superior temporal sulcus of the macaque monkey. J. Neurosci. 6: 145-157.

Sakata, H., H. Shibutani, and K. Kawano (1980) Spatial properties of visual fixation neurons in posterior parietal association cortex of the monkey. J. Neurophysiol. 4.3: 1654-1672.

Sakata, H., H. Shibutani, K. Kawano, and T. L. Harrington (1985) Neural mechanisms of space vision in the parietal association cortex of the monkcy. Vis. Res. 25: 453-463.

Seltzer, B., and D. Pandya (1978) Afferent cortical connections and architectonics of the superior temporal sulcus and surrounding cortex in the rhesus monkey. Brain Res. 149: 1-24.

Seltzer, B., and D. Pandya (1980) Converging visual and somatic sensory cortical input to the intraparietal sulcus of the rhesus monkey. Brain Res. 192: 339-351.

Seltzer, B., and D. N. Pandya (1984) Further observations on parietotemporal connections in the rhesus monkey. Exp. Brain Res. 55:301312

Shipp, S., and S. Zeki (1985) Segregation of pathways leading from 
area V2 to areas V4 and V5 of macaque monkey visual cortex. Nature 315: 322-325.

Sillito, A. M. (1977) Inhibitory processes underlying the directional specificity of simple, complex, and hypercomplex cells in the cat's visual cortex. J. Physiol. (Lond.) 271: 699-720.

Steinmetz, M. A., B. C. Motter, C. J. Duffy, and V. B. Mountcastle (1987) Functional properties of parietal visual neurons: Radial organization of directionalities within the visual field. J. Neurosci. 7: 177-191.

Stephanis, C., and H. Jasper (1964) Intracellular microelectrode studies of antidromic responses in cortical pyramidal tract neurons. $J$. Neurophysiol. 27: 828-854.

Tanaka, K., K. Hikosaka, H. Saito, M. Yukie, Y. Fukada, and E. Iwai (1984) Analysis of the local and wide-field movements in the superior temporal visual areas of the macaque monkey. J. Neurosci. 4: 134 144.

Thurstone, L. L. (1927) A law of comparative judgement. Psychol. Rev. 24: 273-286.

Ungerleider, L. G. (1985) The corticocortical pathways for object recognition and spatial perception. In Pattern Recognition Mechanisms, C. Chagas, ed., pp. 21-37, Pontifical Academy of Sciences, Vatican City.

Ungerleider, L. G., and M. Mishkin (1982) Two cortical visual systems. In Analysis of Visual Behavior, D. J. Ingle, M. A. Goodale, and
R. J. W. Mansfield, eds., pp. 549-587, MIT Press, Cambridge, MA Ungerleider, L. G., R. Desimone, and M. Mishkin (1980) Cortical projections of area MT in the macaque monkey. Soc. Neurosci. Abstr. 8: 680 .

Ungerleider, L. C., R. Gattass, A. P. B. Sousa, and M. Mishkin (1983) Projections of area V2 in the macaque. Soc. Neurosci. Abstr. 9: 152. Van Essen, D. C. (1979) Visual areas of the mammalian cerebral cortex. Annu. Rev. Neurosci. 2: 227-263.

Van Essen, D. C., and J. H. R. Maunsell (1979) Hierarchical organization and functional streams in the visual cortex. Trends Neurosci. 6: 370-375.

Weller, R. E., and J. H. Kaas (1983) Retinotopic patterns of connections of area 17 with visual areas V-II and MT in macaque monkeys. J. Comp. Neurol. 220: 253-279.

Wolbarsht, M. L., E. F. MacNichol, Jr., and H. G. Wagner (1960) Glass insulated platinum microelectrode. Science 132: 1309-1310.

Zeki, S. M. (1971) Convergent input from the striate cortex (area 17) to the cortex of the superior temporal sulcus in the rhesus monkey. Brain Res. 28: 338-340.

Zeki, S. M. (1974) Functional organization of a visual area in the posterior bank of the superior temporal sulcus of the rhesus monkey. J. Physiol. (Lond.) 236: 549-573.

Zeki, S. M. (1978) The cortical projections of foveal striate cortex in the rhesus monkey. J. Physiol. (Lond.) 277: 227-244. 